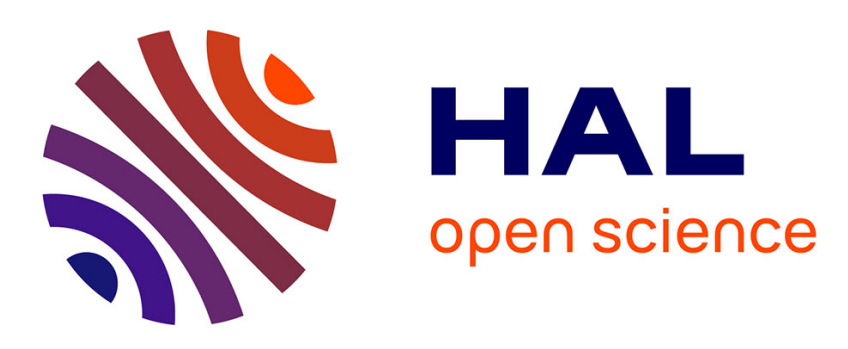

\title{
Unveiling of the mechanisms of acoustic streaming induced by sharp edges
}

Chuanyu Zhang, Xiaofeng Guo, Laurent Royon, Philippe Brunet

\section{To cite this version:}

Chuanyu Zhang, Xiaofeng Guo, Laurent Royon, Philippe Brunet. Unveiling of the mechanisms of acoustic streaming induced by sharp edges. Physical Review E , 2020, 102 (4), 10.1103/physreve.102.043110 . hal-03051910

\section{HAL Id: hal-03051910 https://hal.science/hal-03051910}

Submitted on 10 Dec 2020

HAL is a multi-disciplinary open access archive for the deposit and dissemination of scientific research documents, whether they are published or not. The documents may come from teaching and research institutions in France or abroad, or from public or private research centers.
L'archive ouverte pluridisciplinaire HAL, est destinée au dépôt et à la diffusion de documents scientifiques de niveau recherche, publiés ou non, émanant des établissements d'enseignement et de recherche français ou étrangers, des laboratoires publics ou privés. 


\title{
Unveiling of the mechanisms of acoustic streaming induced by sharp edges
}

\author{
Chuanyu Zhang, ${ }^{*}$ Xiaofeng Guo $\odot{ }^{\dagger}{ }^{\dagger}$ and Laurent Royon \\ Université de Paris, LIED, UMR 8236, CNRS, F-75013 Paris, France \\ Philippe Brunet ${ }^{\S}$ \\ Université de Paris, MSC, UMR 7057, CNRS, F-75013 Paris, France
}

(Received 13 February 2020; accepted 5 October 2020; published 28 October 2020)

\begin{abstract}
Acoustic waves can generate steady streaming within a fluid owing to the generation of viscous boundary layers near walls of typical thickness $\delta$. In microchannels, the acoustic wavelength $\lambda$ is adjusted to twice the channel width $w$ to ensure a resonance condition, which implies the use of MHz transducers. Recently, though, intense acoustic streaming was generated by acoustic waves of a few $\mathrm{kHz}$ (hence with $\lambda \gg w$ ), owing to the presence of sharp-tipped structures of curvature radius at the tip $r_{c}$ smaller than $\delta$. The present study quantitatively investigates this sharp-edge acoustic streaming via the direct resolution of the full Navier-Stokes equation using the finite element method. The influence of $\delta, r_{c}$, and viscosity $v$ on the acoustic streaming performance is quantified. Our results suggest choices of operating conditions and geometrical parameters, in particular the dimensionless tip radius of curvature $r_{c} / \delta$ and the liquid viscosity.
\end{abstract}

DOI: 10.1103/PhysRevE.102.043110

\section{INTRODUCTION}

Acoustic streaming (AS) is a time-averaged steady flow generated by an acoustic field in a fluid, due to secondorder nonlinear effects originating from the coupling between acoustics and hydrodynamics. The phenomenon has attracted the interest of researchers for almost two centuries, since as early as 1831 when Faraday [1] first observed steady patterns of light particles on vibrating plates. More recently, AS has been proven to be a useful and noninvasive solution in various applied situations [2], like mixing under low-Reynolds-number laminar flow conditions [3], particles manipulation and sorting [4-9], particles patterning [10,11], or heat transfer $[12,13]$.

Acoustic streaming originates from the dissipation of acoustic energy within a fluid, which creates a time-averaged effective forcing [2,14-22]. Meanwhile, depending on where in the fluid acoustic attenuation is mainly prevalent, AS can be induced either by viscous bulk fluid attenuation, denoted as Eckart streaming [15,17], or by the generation of viscous boundary layers (VBL) along walls, denoted as Rayleigh-Schlichting streaming [2,18-22]). For the latter, the development of an unsteady VBL can lead to nonzero timeaveraged Reynolds stress inside this layer [19]. Rayleigh's theory $[18,19,22]$ describes that intense vorticity generated within the VBL appears as an array of eddies pairs (called inner vortices) aligned along the channel walls [7,23,24]. This stress extends its influence beyond the VBL of thickness $\delta=$

\footnotetext{
*chuanyu.dream@gmail.com

${ }^{\dagger}$ Also at Université Gustave Eiffel, ESIEE Paris, F-93162, Noisy le Grand, France.

†laurent.royon@univ-paris-diderot.fr

§philippe.brunet@univ-paris-diderot.fr
}

$\left(\frac{2 v}{\omega}\right)^{\frac{1}{2}}$ from the wall, where $v$ is the liquid kinematic viscosity and $\omega$ is the angular frequency of the wave, and induces larger-scale eddies of typical width $\lambda / 2$ half of the acoustic wavelength $[23,25]$ in the fluid bulk.

To achieve AS in microfluidics geometries, taking here the usual definition that the system size is inferior to $1 \mathrm{~mm}$ in at least one dimension, the channel width $w$ and the wavelength are generally adjusted to ensure a resonance condition, typically obtained when $w \simeq \lambda / 2$ [26]. Given that the sound velocity in water and in most liquids ranges between 1000 and $1800 \mathrm{~m} / \mathrm{s}$, the wave frequency $f$ shall then be of the order of a few MHz. Therefore, while typical cost-effective transducers and associated amplifiers are generally in a range of a few $\mathrm{kHz}$ to a few tens of $\mathrm{kHz}$, they should in principle fail to generate AS in microchannels, as the acoustic field would then be homogeneous in space. Although a few studies could circumvent this limitation by tuning the excitation of immersed bubbles [27], by using micropillars [28] or flexural waves on a flexible wall [13], by prescribing a wavy channel geometry [29-31], or by tuning streaming modes within the transducer plane [32], the majority of them were carried out under ideal geometries such as infinite or semi-infinite domains. Still, remaining issues concern the influence of geometry, for instance the presence of obstacles or nonstraight profiles like constrictions, or in situations of confinement when $\delta$ can be comparable to one of the channel dimensions [25].

Recent studies have shown that intense AS could be generated via the coupling between acoustic waves and sharp structures [33-36]. One of the particularities and main advantages of "sharp-edge AS" is that it is generated at relatively low frequency, typically in the $\mathrm{kHz}$ range. Meanwhile, the order of magnitude of the steady streaming velocity can even be comparable to the vibration velocity, hence up to several hun- 

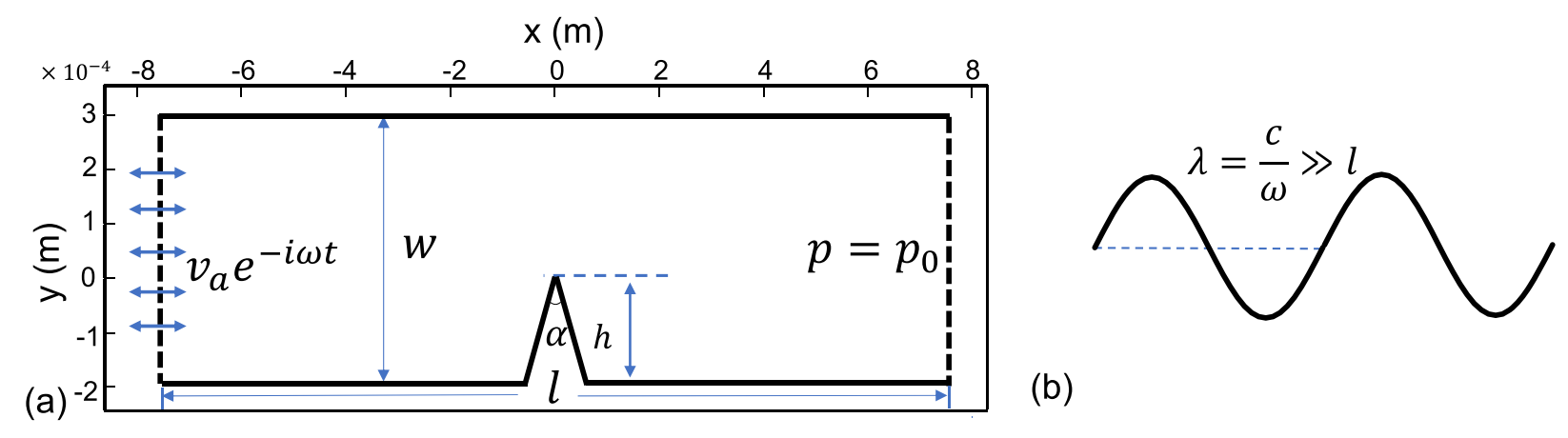

(b)

(c)
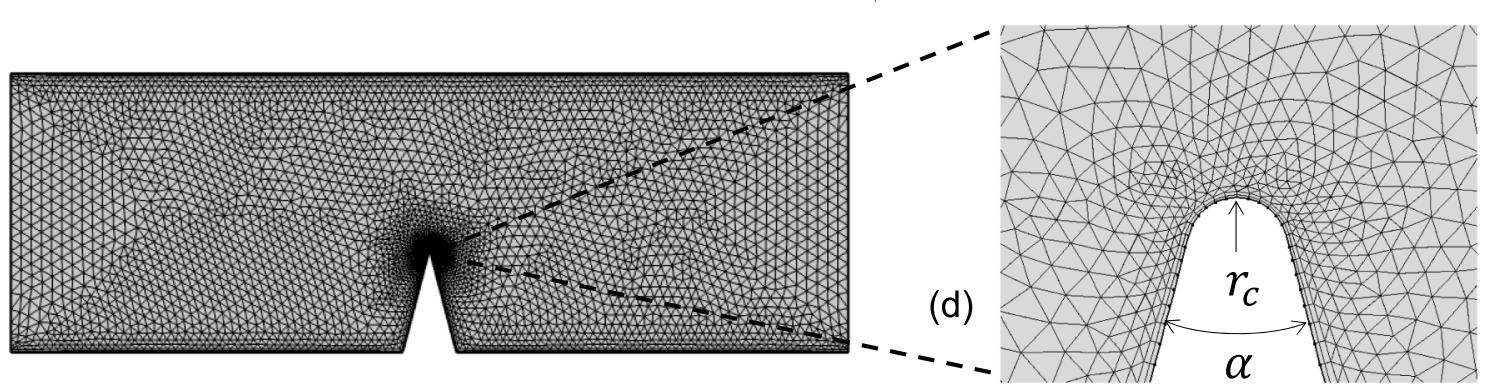

FIG. 1. The geometry of the domain of study is sketched in the top-left inset, together with the acoustic wave parameters in the top-right inset. The mesh for the computation is shown in the bottom figure, with a magnified view around the sharp edge tip on the right.

dreds of $\mathrm{mm} / \mathrm{s}$ [37]. Benefiting from this strong disturbance within the fluid, various applications using sharp structures streaming have been developed in microfluidics: mixing processes [35,38], bioparticle control [39,40], as well as various on-chip devices [34,41].

However, until now, the underlying mechanisms of this streaming are not yet fully clear [42]. First, the pioneering study from T. J. Huang's group [33] attributes the induced streaming flow to the mechanical vibrations of the sharp structures induced by a transducer stuck on the microchannel wall. Such a vibration was indeed observed with high-speed imaging, and it raises the question of the adaptation of the sharp edge geometry to the prescribed frequency in order to ensure a resonance condition. In Zhang et al.'s study [37], an oscillating flow was prescribed to the whole fluid, which also generates strong streaming around the sharp tip but without the constraint of operating at a specific frequency. Although Ovchinnikov et al's study [42] suggests that both situations should in principle lead to similar streaming flows, the first-order fluid oscillations in both situations should be different from each other. A very recent study proposed more complete analytical expressions for the streaming flow, as well as detailed flow profiles from numerics and experiments at much higher frequency [43]. The same authors evidenced that sharp-edged structures are also suitable for particle clustering via acoustic radiation pressure [44].

Second, although both experiments [33,37] and simulations [35,42] confirm the AS intensity depends on the sharpness of the tip, none of them dissociates the tip angle $\alpha$ from the curvature diameter $2 r_{c}$, as shown in Figs. 1(a) and $1(d)$, both of which being a sign of sharpness. The difficulty is that in practice, the microlithography techniques make these two quantities related to each other [37]. Therefore, numerical simulations could tackle this challenging question. Third, while most studies on acoustic streaming generated around obstacles concern situations where $\delta \ll 2 r_{c}$ and those by Ovchinnikov et al. [42] and by Doinikov et al. [43] address the opposite situation $\left(\delta \gg 2 r_{c}\right)$, it is unclear how the crossover between the two situations takes place. Very recently published experimental results showed that the dependence on viscosity and frequency can be more complex than expected even if $\delta \gg 2 r_{c}$ [45], possibly as a consequence of a constrained geometry as $\delta$ can be of the same order as the channel depth $d$.

Finally, from a theoretical point of view, sharp-edge AS remains a case study for a nonlinear framework in acoustofluidics equations. Indeed nonlinear terms coupling both the steady and periodic velocity fields can become dominant, or at least nonnegligible, a feature which in turn is susceptible to make the classical perturbation theory no longer adapted. This situation is the consequence of that, as mentioned above, the streaming velocity can be locally as strong as the vibration velocity [37].

Motivated by these unsolved questions, and in the aim to propose quantitative predictions, the current study addresses the AS flow under different operating conditions (vibration amplitude $A$, sound frequency $f$ ), fluid properties (kinematic viscosity $\nu$ ), and geometries (tip sharpness quantified by both $r_{c}$ and $\alpha$ ). This parametric study is made possible by directly solving the full Navier-Stokes equation using finite elements method. Results from the direct numerical simulation (DNS) are first validated by recent experiments, and then compared with those from simulations using classical perturbation theory (PT). This comparison points out the necessity to treat and include all nonlinear terms in the numerical model. In a more applied purpose, this study aims to provide a framework for designing the optimal geometrical structure which would provide the strongest possible AS flow field for a given acoustic forcing. 


\section{THEORETICAL MODEL}

\section{A. Equations of motion}

The fundamental equations governing acoustic streaming have been previously presented in various theoretical studies $[2,14,16,20,21,46,47]$, which we summarize thereafter. Bold and normal font style respectively represent vectorial and scalar quantities. Without external body forces nor heat sources and for an isotropic homogeneous fluid, the mass and momentum conservation equations governing the flow are as follows:

$$
\begin{gathered}
\frac{\partial \rho}{\partial t}+\nabla \cdot(\rho \mathbf{v})=0 \\
\rho \frac{\partial \mathbf{v}}{\partial t}+\rho(\mathbf{v} \cdot \nabla) \mathbf{v}=\nabla \cdot \overline{\bar{\sigma}}
\end{gathered}
$$

where $\rho$ is the liquid density and $\mathbf{v}$ the velocity field. The Cauchy stress tensor $\overline{\bar{\sigma}}$ is the sum of the viscosity (v) term $\overline{\bar{\tau}}$ and pressure term $-p \overline{\bar{I}}$. As in our situation, $\lambda \gg w$, and that the Mach number $\mathrm{Ma}=v_{a} / c \ll 1$, the fluid can be treated as being incompressible, leading to $\overline{\bar{\sigma}}=-p \overline{\bar{I}}+\mu\left(\nabla \mathbf{v}+\nabla \mathbf{v}^{\top}\right)$. Then Eqs. (1) and (2) can be reduced to:

$$
\begin{gathered}
\nabla \cdot \mathbf{v}=0 \\
\rho \frac{\partial \mathbf{v}}{\partial t}+\rho(\mathbf{v} \cdot \nabla) \mathbf{v}+\frac{1}{\rho} \nabla p=\mu \nabla^{2} \mathbf{v} .
\end{gathered}
$$

To analyze the AS flow, the PT constitutes a common framework [2,14,16,20,21,46,47]. The velocity and pressure fields are decomposed into an unperturbed state, oscillating and steady streaming parts, hereafter denoted with subscripts $0, \omega$, and $s$, respectively:

$$
\begin{aligned}
& \mathbf{v}=\mathbf{v}_{0}+\mathbf{v}_{\omega}+\mathbf{v}_{s}, \mathbf{v}_{\omega}=\operatorname{Re}\left(\mathbf{v}_{a} e^{i \omega t}\right), \\
& p=p_{0}+p_{\omega}+p_{s}, p_{\omega}=\operatorname{Re}\left(p_{a} e^{i \omega t}\right),
\end{aligned}
$$

where $\mathbf{v}_{0}=\mathbf{0}$ is the unperturbed bulk flow considered to be null in this study, $\mathbf{v}_{\omega}$ is the acoustic (oscillating) part of the velocity field, $\mathbf{v}_{a}$ is the complex amplitude of the vibration velocity, and $\mathbf{v}_{s}$ is the steady streaming velocity; similarly, $p_{\omega}, p_{a}$ are the pressure and complex amplitude of the acoustic pressure field, $p_{0}$ is the gauge atmospheric pressure and $p_{s}$ is the steady pressure field associated to the streaming flow. Similar notations can be used for the density, in the situation where compressible effects would be taken into account. The classical PT assumes $\left\|\mathbf{v}_{s}\right\| \ll\left\|\mathbf{v}_{\omega}\right\|$ and $p_{s} \ll p_{\omega}$, i.e., that the streaming flow velocity is of considerably lower magnitude than the driving acoustic velocity [2,15,35,36,42,43,46-49]. Given the strong AS which is generated near sharp edges in our study, we here dismiss these simplifying assumptions.

By injecting the decomposition of Eq. (5) into Eqs. (3) and (4), and after a bit of algebra, the momentum equation leads to time-dependent [Eq. (6)] and steady [Eq. (7)] parts:

$$
\begin{aligned}
i \omega \mathbf{v}_{a}+\left(\mathbf{v}_{s} \cdot \nabla\right) \mathbf{v}_{a}+\left(\mathbf{v}_{a} \cdot \nabla\right) \mathbf{v}_{s} & =-\frac{1}{\rho} \nabla p_{a}+v \nabla^{2} \mathbf{v}_{a}, \\
\left(\mathbf{v}_{s} \cdot \nabla\right) \mathbf{v}_{s}+\frac{1}{2}\left\langle\operatorname{Re}\left[\left(\mathbf{v}_{a} \cdot \nabla\right) \mathbf{v}_{a}^{*}\right]\right\rangle & =-\frac{1}{\rho} \nabla p_{s}+v \nabla^{2} \mathbf{v}_{s} .
\end{aligned}
$$

Equations (6) and (7) both contain nonlinear terms in velocity, coupling the unsteady and steady components. By time-averaging Eq. (7), one then sets a body force $\mathbf{F}_{\mathbf{s}}$ to account for the nonlinear effects of vibration motions [15,42]:

$$
\left(\mathbf{v}_{s} \cdot \nabla\right) \mathbf{v}_{s}=\frac{1}{\rho}\left(\mathbf{F}_{s}-\nabla p_{s}\right)+v \nabla^{2} \mathbf{v}_{s},
$$

where the body force is as follows:

$$
\mathbf{F}_{s}=-\frac{\rho}{2}\left\langle\operatorname{Re}\left[\left(\mathbf{v}_{a} \cdot \nabla\right) \mathbf{v}_{a}^{*}\right]\right\rangle,
$$

here the operator $\langle$.$\rangle stands for a time-averaging over one$ period of acoustic oscillation $1 / f$.

In the PT framework, the nonlinear terms at the left-hand side of Eq. (6), coupling $\mathbf{v}_{a}$ and $\mathbf{v}_{s}$, are commonly neglected. Also in Eqs. (7) and (8), $\left(\mathbf{v}_{s} \cdot \nabla\right) \mathbf{v}_{s}$ is considered as a negligible, fourth-order term in most previous studies of acoustic streaming [2,15,42,43,46-48]. As stated above, in the case of sharp-edge streaming, ignoring these terms should deviate the modelled results from reality. The primary reason is, as previously mentioned, $\mathbf{v}_{s}$ can be of the same order as $\mathbf{v}_{a}$. It implies that the convection of the acoustic field by the streaming one becomes significant, as it was directly revealed by our previous experimental results, see inset of Fig. 5 in Ref. [37], especially in the upper range of acoustic velocity. The second reason lies in the boundary layer. Under usual situations where $\delta$ is much thinner than any other lengths of the problem-in particular, much smaller than the radius of curvature of the boundary walls, the resolution is carried out by solving separately the streaming flow within the steady VBL $[20,47,48]$ and that outside of the VBL. It consists of prescribing a distribution of slip velocities along walls, previously derived from the calculation within the VBL, to the fluid bulk. In the case of sharp edges when $r_{c}<\delta$, the direct numerical resolution in the whole domain, and especially within the VBL, becomes necessary. Ovchinnikov et al.'s study [42] was dedicated to this situation, and our study is partly inspired by their approach. As our study investigates streaming flows in an extended range of amplitude, we choose to keep these terms in our simulations.

\section{B. Qualitative view of the streaming force}

Let us now briefly examine the term $\mathbf{F}_{s}$ of Eq. (9). We assume that $\mathbf{v}_{a}=\left[\begin{array}{lll}v_{a x} & v_{a y} & 0\end{array}\right]$ is a vector remaining in the $(x y)$ plane, which is true far from the upper and lower walls. Let us then calculate $\mathbf{v}_{a}$ in this plane:

$$
\left(\mathbf{v}_{a} \cdot \nabla\right) \mathbf{v}_{a}=\left(\begin{array}{c}
v_{a x} \frac{\partial v_{a x}}{\partial x}+v_{a y} \frac{\partial v_{a x}}{\partial y} \\
v_{a x} \frac{\partial v_{a y}}{\partial x}+v_{a y} \frac{\partial v_{a y}}{\partial y} \\
0
\end{array}\right) .
$$

Results from our previously reported direct high-speed visualization [37] showed that, near sharp edges, the acoustic velocity field in fluid is aligned in parallel to the nearest wall. Furthermore, the no-slip boundary condition sets $\mathbf{v}_{a}=\mathbf{0}$ along walls so that the amplitude of acoustic oscillations decreases to zero approaching the wall. This velocity gradient is the origin of shear stress within the VBL.

In summary, gradients of acoustic velocity should originate from at least two effects: (i) the no-slip boundary condition which creates variation of acoustic velocity amplitude from $v_{a}=0$ at the wall to $v_{a} \simeq A \omega$ at a distance to the wall farther 
than $\delta$, here $A$ is the spatial amplitude of the acoustic vibration, and (ii) the orientation of $\mathbf{v}_{a}$ bending by an angle of $\pi-\alpha$ over a distance of $2 r_{c}$.

Along a straight horizontal wall, $v_{a y}$ is null and $v_{a x}$ is invariant with $x$. Therefore, only $v_{a x}$ and $\frac{\partial v_{a x}}{\partial y}$ take nonzero values, which implies that $\mathbf{F}_{s}$ is null along a straight wall. This can easily be generalized along any straight wall of arbitrary orientation. However, the streaming force $\mathbf{F}_{s}$ is nonzero where there is a steep change of orientation of $\mathbf{v}_{a}$, typically achieved near a sharp tip. This nonzero force originates from a centrifugal-like effect emphasized in previous studies [37,42].

Let us finally remark that we deliberately choose to keep dimensional quantities in this study. First, our study aims for a quantitative comparison with previous experiments, which is made easier with dimensional quantities. Second, our problem involves four length scales which must be decoupled from each other. More specifically, the acoustic wavelength $(\lambda)$, the channel width $(w)$, the VBL thickness $(\delta)$, and the tip radius of curvature $\left(r_{c}\right)$ must fulfill the condition $\lambda \gg w \gg \delta \gg r_{c}$. This condition would lead to complex formulations for dimensionless equations. Third, the COMSOL software we use in our simulations, naturally handles dimensional quantities.

\section{DESCRIPTION OF THE NUMERICAL SCHEME}

Most of the numerical results presented in this paper are based on the direct solving of the Navier-Stokes equation (DNS). We also present a few results obtained from the PT inspired from Ovchinnikov et al.'s study [42] so that the efficiency of the two methods can be compared under different conditions. Both PT and DNS simulations are conducted with finite element method (FEM) using COMSOL Multiphysics [50]. Details of the simulation implementation scheme are described in Appendices A to E.

\section{A. Domain of study}

The geometry of the microchannel with a single sharp tip are detailed in Fig. 1. Length and width of the channel are respectively $l=1.5 \mathrm{~mm}$ and $w=0.5 \mathrm{~mm}$. A symmetrical sharp structure with a tip angle $\alpha$ and a curvature diameter $2 r_{c}$ is located on one side of the channel. While both $\alpha$ and $2 r_{c}$ are taken at different values for different simulation cases, the height of the sharp structure is kept constant: $h=0.18 \mathrm{~mm}$.

It worth noting that the simulations are conducted in the framework of a bidimensional (2D) geometry. Precisely, the channel is considered infinitely deep. This choice is justified by two main reasons. First, all previous experiments of sharpedge streaming, including ours, are conducted with water $(\rho=$ $1000 \mathrm{~kg} / \mathrm{m}^{3}$ and $v=10^{-6} \mathrm{~m}^{2} / \mathrm{s}$ at $20^{\circ} \mathrm{C}$ ) and $f$ equal to a few $\mathrm{kHz}$, yielding $\delta$ between 8 and $15 \mu \mathrm{m}$, while the channel depth $d$ is equal or larger than $50 \mu \mathrm{m}$. Second, the cross-sectional depth:width aspect ratio is roughly $1: 10$. As a consequence, the streaming develops essentially within the $(x y)$ plane.

Near the sharp edge, the mesh is refined (Fig. 1) since velocity gradients, and thus the streaming force, are expected to be locally concentrated near the tip, since the mesh step is locally much smaller than $r_{c}$. The mesh refining also allows to accurately account for the sharp geometry of the tip. Furthermore, a similar mesh refinement is also imposed within the
VBL of both channel and sharp-edge walls. This is essential to finely simulate the effect of viscous shear stress from which AS originates.

\section{B. Boundary conditions}

DNS is different from the PT method as it directly computes the fluid motion equations (6)-(8) for the acoustic oscillations (time-periodic) and for the steady streaming. Periodic boundary conditions are set at left and right ends of the channel. The left end (here set as the inlet) is attributed a periodic velocity $v_{x}=v_{a} \sin (2 \pi f t)$ along the horizontal direction and $v_{y}=0$ along the vertical one. For the right end (outlet), a condition of pressure fixed at $p_{0}$ is assigned (See Fig. 1(a)).

Since the fluid remains incompressible, and the length scale of the domain is much smaller than the acoustic wavelength $(l \ll \lambda)$, the above conditions result in an inphase periodic velocity for the right and left borders. These conditions are supported by experimental observations of oscillations of fluid particles within the whole channel, while the sharp-edged tip remains static in the laboratory frame [37]. A no-slip condition is prescribed on all other channel boundaries including along the sharp edge itself.

For the time-dependent simulations, each acoustic period is discretized into 50 time steps, for an overall duration of 30 acoustic periods. It turns out that this duration is sufficient to allow the full establishment of a quasisteady acoustic streaming, once the flow is averaged over an acoustic period. Moreover, the choice of 50 time steps per acoustic period is validated by comparing the streaming results from four different time steps. This validation process is documented in Appendix D and shown in Fig. 13.

\section{RESULTS AND DISCUSSIONS}

\section{A. Validation of the numerical scheme}

The comparison between previous experimental results [37] and present DNS ones ensures the validation of our numerical scheme. Figures 2 and 3 intend to illustrate the mechanism of acoustic streaming by showing both typical acoustic and steady velocity fields. Figure 2(a) presents a qualitative sketch and Figs. 2(b) and 2(c) show typical amplitude and orientation of the acoustic velocity field from both experiments and numerical simulations.

\section{Acoustic velocity}

As shown in Fig. 2(a) and Figs. 3(a)-3(d), the acoustic field takes place in the whole channel. Far from the walls, fluid particles oscillate with fixed amplitude $A$ and with orientation roughly imposed by that of the nearest wall. As previously stated, while for $\lambda \gg(w, l)$ no streaming force can develop within the microchannel, the presence of a tip induces a sharp spatial variations in the orientation of vibrations, see Fig. 2(c), where the aforementioned centrifugal effect clearly appears in the vicinity of the tip. This effect induces a locally strong streaming jet shooting from the tip, as shown in Fig. 3(b).

Careful high-speed particle image velocimetry (PIV) measurements of the acoustic flow reveal that oscillations close to the tip are stronger than elsewhere in the channel, roughly by a factor of two to three. The exact value of this factor is 
(a)

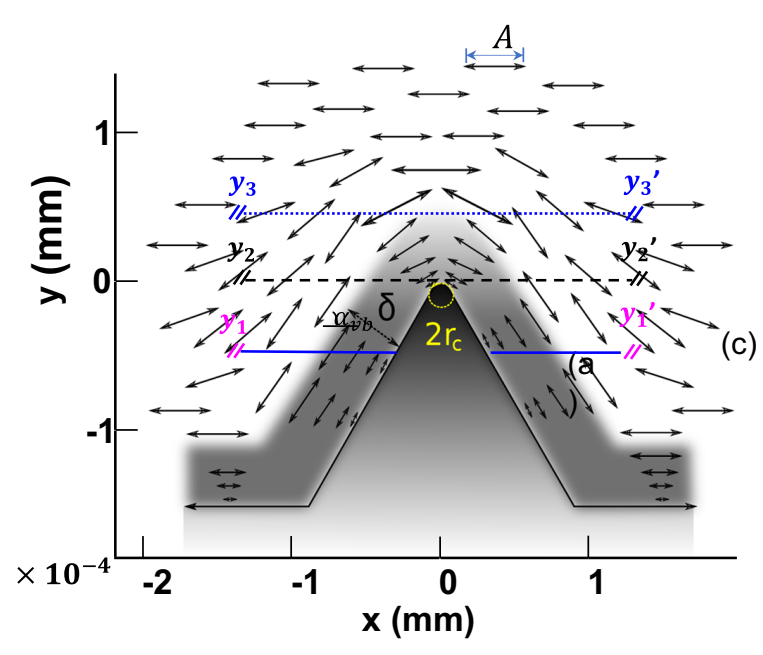

(b)
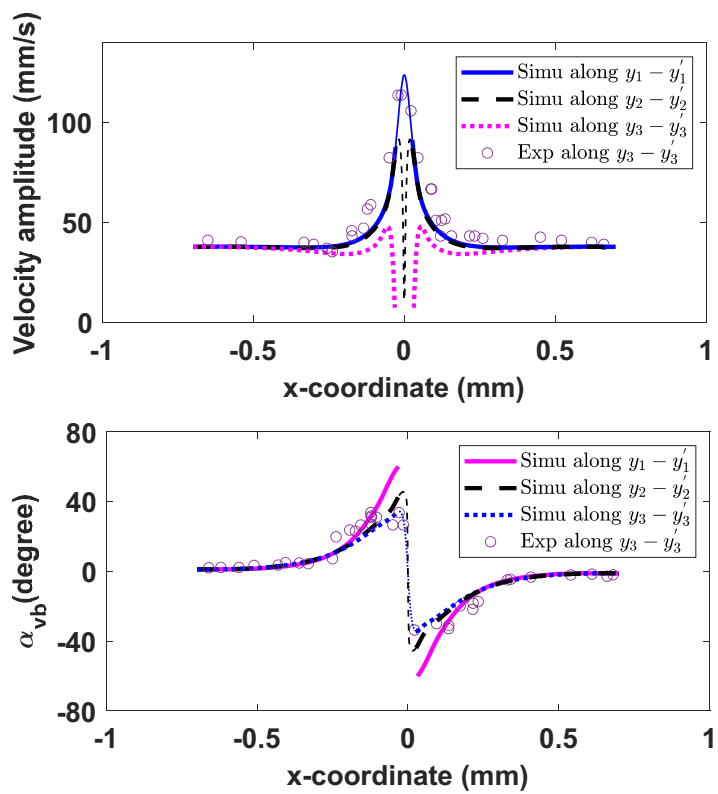

FIG. 2. The acoustic vibration and streaming flow are shown around the sharp edge structure. (a) Sketch of the acoustic vibrations of fluid particles near the sharp edge, $\delta$ is the acoustic boundary layer, the segment $y_{1}-y_{1}^{\prime}$ is located 0.05 mm below the tip; $y_{2}-y_{2}^{\prime}$ intersects the tip; $y_{3}-y_{3}^{\prime}$ is located $0.01 \mathrm{~mm}$ above the tip. (b) Amplitude of the vibration velocity recorded along $y_{1}-y_{1}^{\prime}, y_{2}-y_{2}^{\prime}$, and $y_{3}-y_{3}^{\prime}$. Circles stand for experiments recorded along $y_{3}-y_{3}^{\prime}$. (c) Orientation of the vibration velocity $\alpha_{v b}=\arctan \left[v_{a y} / v_{a x}\right]$ along $y_{1}-y_{1}^{\prime}, y_{2}-y_{2}^{\prime}$, and $y_{3}-y_{3}^{\prime}$. Circles stand for experiments recorded along $y_{3}-y_{3}^{\prime}$. Parameters: $\alpha=60^{\circ}, 2 r_{c}=5.8 \mu \mathrm{m}, f=2500 \mathrm{~Hz}, v_{a}=37.8 \mathrm{~mm} / \mathrm{s}$, and $\delta=11.5 \mu \mathrm{m}$.

found to depend on both $\alpha$ and $v_{a}$, and presumably on the height $h$.

Figures 2(b) and 2(c) respectively show the amplitude of the acoustic velocity $v_{a}$ and the vibration orientation, quantified by the angle $\alpha_{v b}$, obtained from both experiments and simulations. Approaching $x=0$, the velocity $v_{a}$ sharply increases from its value far from the tip $(38.5 \mathrm{~mm} / \mathrm{s})$, to reach its maximum value at $x=0$ (here roughly $120 \mathrm{~mm} / \mathrm{s}$ ) and then sharply decreases back to its value at infinity, see Fig. 2(b). The values of the velocity amplitude $v_{a}$ and angle $\alpha_{v b}$ are respectively symmetrical and antisymmetrical about $x=0$, along the vertical direction from the tip. For both quantities, the influence of the sharp structure is significant mainly within the region from $x=-0.2$ to $0.2 \mathrm{~mm}$, hence comparable to the height of the structure $h=0.18 \mathrm{~mm}$.

As shown in Fig. 2(c), the orientation angle $\alpha_{v b}$ of $\mathbf{v}_{a}$ varies along the $x$ direction. The evolution of $\alpha_{v b}(x)$ depends much on the distance from the tip $y$. If $y=0.01 \mathrm{~mm}$ (line $\left[y_{3}-y_{3}^{\prime}\right]$ ), hence roughly equal to $\delta, \alpha_{v b}$ increases from 0 far enough from the tip, up to roughly $32^{\circ}$. Then it sharply decreases down to its corresponding negative value, roughly $-32^{\circ}$, continuously and slowly increases back to zero far away from the tip. This profile is in very good agreement with our previous measurements obtained from high-speed imaging [37] and extracted at the same distance $y$ from the tip. In Fig. 2(c), we also plot $\alpha_{v b}(x)$ along the line $\left[y_{2}-y_{2}^{\prime}\right]$, which corresponds to $y=0$, hence intersecting the edge right at the tip. The overall profile of $\alpha_{v b}(x)$ resembles the previous one, except near the tip where the maximal and minimal values have larger absolute values, around $40^{\circ}$ and $-40^{\circ}$, respectively. Finally, the values extracted from a line $\left[y_{1}-y_{1}^{\prime}\right]$ lower than the tip, show the same trend for $\alpha_{v b}(x)$, with maximal and minimal values very close to that of the wall, i.e., $(\pi / 2-\alpha / 2)$ and $(\alpha / 2-\pi / 2)$.

At this stage and at a qualitative level, we can conclude that the value of $\alpha$ sets the amplitude of the jump in the orientation of $\mathbf{v}_{a}$, whereas the value of $r_{c}$ sets the sharpness of the spatial variation of orientation. Both of them should play crucial influence on the magnitude of acoustic perturbation within the fluid.

\section{Streaming velocity}

Figure 3(e) shows the steady streaming velocity and corresponding streamlines. We observe perfectly symmetrical streaming vortices in the vicinity of the sharp tip. This clearly shows how focused the driving streaming force is, in particular in the vicinity of the sharp tip, and confirms previous findings [42]. Thereafter, we denote the maximal value, evaluated within the whole streaming flow, as $v_{\text {sm. }}$. In sharp-edge streaming, the velocity is found to be maximal along the $y$ axis, hence at $x=0$, and directed toward the $y$ direction. We shall see that this is no longer the case when $r_{c}$ is large enough with respect to $\delta$.

Figure 4 shows the streaming velocity $v_{s y}(x=0, y)$ along the $y$ direction, with the frame origin $(x=0$ and $y=0)$ taken at the tip. For a reason of symmetry, $v_{s y}(x=0, y)$ is oriented along $y$ so that only the $y$ component of $v_{s}$ is plotted. Results from DNS are in very good agreement with experiments extracted from our previous study [37]. In addition, the numerical study further allows to access velocity within the thin VBL, which was hardly possible in experiments, due to limitations of the visualization technique. Within the VBL range $y \leqslant \delta$, the streaming velocity sharply increases with $y$ to its maximum value $v_{\text {sm }}$ obtained near $y \simeq \delta$. Beyond this 

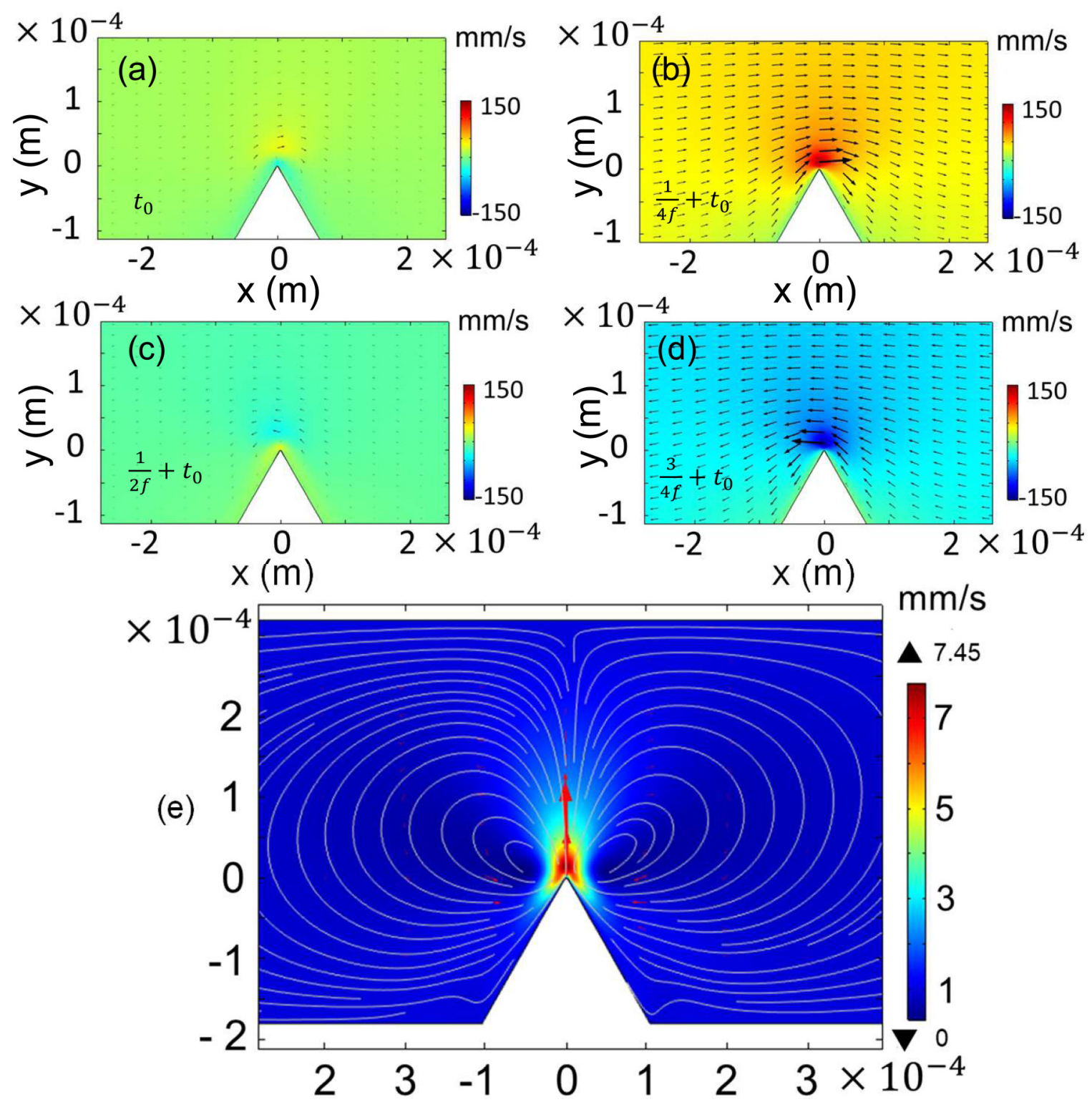

FIG. 3. The acoustic vibration and streaming flow are shown around the sharp edge: [(a)-(d)] Successive velocity fields at different time (or phase) during an acoustic period, (e) Magnitude and streamlines of the streaming flow, from time-average during several acoustic periods. Parameters: $\alpha=60^{\circ}, 2 r_{c}=5.8 \mu \mathrm{m}, f=2500 \mathrm{~Hz}, v_{a}=37.8 \mathrm{~mm} / \mathrm{s}, \delta=11.5 \mu \mathrm{m}$.

point, the streaming velocity decreases along the $y$ direction and vanishes to zero at a distance from the tip roughly equal to $w-h$, here $\simeq 0.3 \mathrm{~mm}$.

We also define $v_{\mathrm{sm}}^{\prime}$ as the maximal streaming velocity determined only on the $y$ axis. Let us here point out that for most situations investigated in this study, namely the situation of sharp edge where $2 r_{c}<\delta, v_{\text {sm }}$ is found to be along the $y$ axis (at $x=0$ and $y \simeq \delta$ like in Fig. 4), and then $v_{\mathrm{sm}}=v_{\mathrm{sm}}^{\prime}$. However, when $r_{c}$ is significantly larger than $\delta$, the maximal velocity is found out of the $y$ axis, typically in the periphery of the two eddies of the VBL, making $v_{\mathrm{sm}}$ different from $v_{\mathrm{sm}}^{\prime}$. This is illustrated by the two insets of Fig. 4. In the latter situation, these two values shall be treated separately. Let us finally remark that the ratio $r_{c} / \delta$ rules the crossover between sharp-edge and classical Rayleigh streaming.

\section{DNS versus PT}

Based on the above analyses, we extract $v_{\text {sm }}$ as a relevant quantity to characterize the streaming velocity field, under the combination of different operating parameters. Other quantities like the size of streaming vortices and the area influenced by the streaming flow are directly related to $v_{\text {sm }}$ [37]. In order to better quantify the situations where $r_{c}>\delta$, and in particular to understand and quantify the crossover between sharp-edge and smooth-edge configurations, we also systematically extract $v_{\mathrm{sm}}^{\prime}$, hence restricting the area to the $y$ axis.

First, we quantitatively investigate the influence of the forcing on $v_{\mathrm{sm}}$. Figure 5 shows a quadratic dependence between $v_{\text {sm }}$ and the acoustic velocity amplitude $v_{a}$. Experimental results with water are taken from Ref. [37] and from three sets of values of $\alpha$ and $r_{c}$. Results from DNS and PT simulation are shown respectively as plain and dashed lines for the three sets of parameters. At low-enough acoustic amplitude, both 


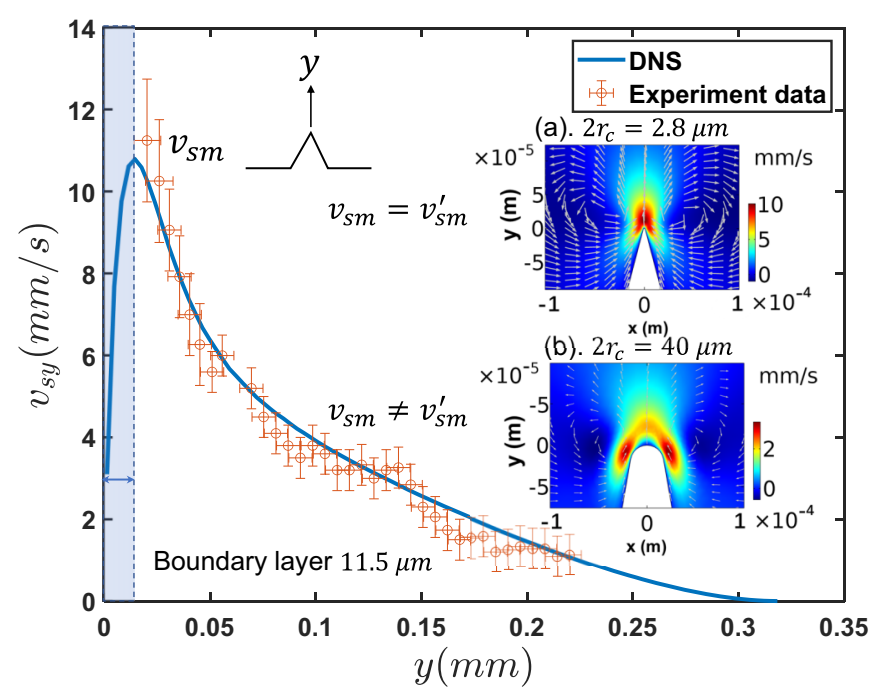

FIG. 4. The streaming velocity profile is plotted along the $y$ direction, with comparison between experiments and simulations. Conditions are $\alpha=30^{\circ}, 2 r_{c}=2.8 \mu \mathrm{m}$, and $v_{a}=37.8 \mathrm{~mm} / \mathrm{s}$. Due to the finite size of PIV particles, the flow could not be solved within the boundary layer with a thickness of $11.5 \mu \mathrm{m}$. The two inserted maps show the magnitude of the streaming velocity field (reddish color standing for larger velocity) and its direction (arrows) in respectively two situations: Sharp edge situation [upper map (a)] where the maximal velocity $v_{\mathrm{sm}}$ is located on the $y$ axis and round edge one [lower map (b)] where $v_{\mathrm{sm}}$ is located besides the $y$ axis and hence different from $v_{\mathrm{sm}}^{\prime}$.

the PT and DNS simulations give satisfactory agreement with experiments.

However, at larger acoustic velocity, results of DNS are in better agreement with experiments than those from PT. The latter tends to overestimate the streaming velocity by roughly $20 \%$ under strong acoustic vibration.

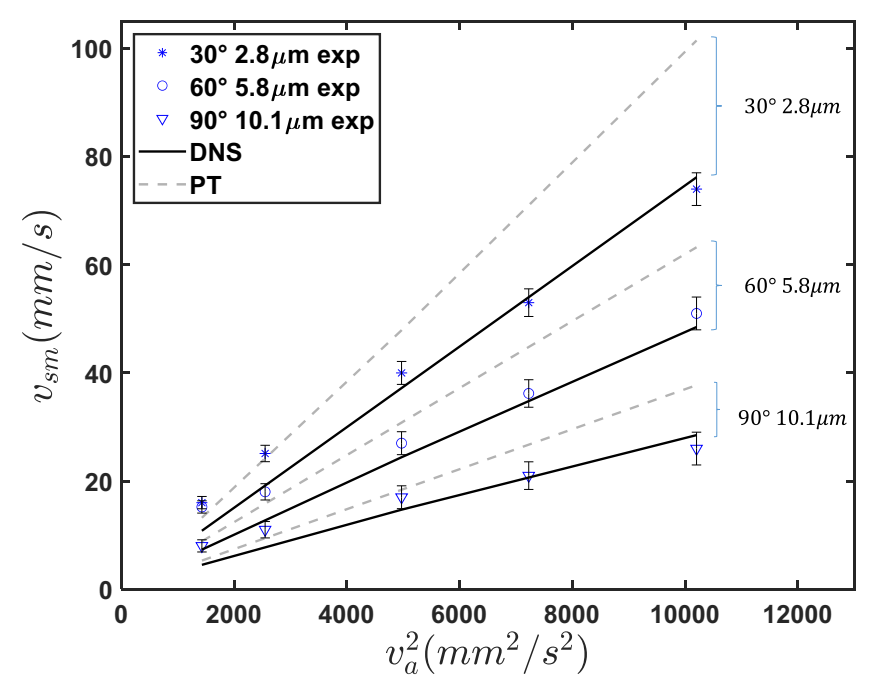

FIG. 5. The maximum streaming velocity is plotted versus the square of acoustic vibration $v_{a}^{2}$ for different sets of values for angle $\alpha$ and radius of curvature $r_{c}$. Results are extracted from experiments (symbols), PT simulation (dashed lines), and DNS (plain lines).
The above results suggest that DNS provides a better prediction of the streaming velocity around the tip and it can be considered as a reliable method to predict the streaming flows generated by sharp structures.

\section{B. Quantitative results \\ 1. Vorticity maps}

Figure 6 show vorticity maps of the streaming flow, calculated by DNS with different tip angle $\alpha$ and curvature diameter $2 r_{c}$. The acoustic forcing velocity is taken relatively strong, at $v_{a}=101.7 \mathrm{~mm} / \mathrm{s}$, corresponding to the right uttermost points in Fig. 5. It reveals that intense vorticity is localized near the tip, within the VBL and takes values of opposite signs in the regions to the left and right of the tip. The inner vortices in turn induce outer vortices of opposite sign and of larger size, further away from the tip [see in particular Figs. 5(a) and 5(b)]. These outer vortices correspond to the ensemble of streamlines shown in Figs. 3(b). For all cases, the extrema of vorticity roughly remain at the same locations: very close to the tip and at each side of it.

Figures 6(a)-6(d) illustrate the comparative influence on vorticity maps of the different angle $\alpha$, ranging from acute $\left(\alpha=12^{\circ}\right)$ to obtuse $\left(\alpha=120^{\circ}\right.$ ), while keeping $r_{c}$ constant. More intense vorticity appears for sharper structures [see Figs. 6(a) and 6(b)] while its magnitude decreases as $\alpha$ increases [see Figs. 6(c) and 6(d)]. Figures 6(e)-6(h) illustrate the influence of the value of $r_{c}$, while keeping $\alpha$ constant. Two distinct behavior emerge: Within the range $2 r_{c}>\delta$ [Figs. $6(\mathrm{~g})$ and $6(\mathrm{~h})]$, increasing $r_{c}$ leads to more spread and weaker vortices, while in the range $2 r_{c}<\delta$ [Figs. 6(e) and 6(f)], vorticity does not vary significantly with $r_{c}$. These two latter cases show that the flow is weakly influenced by $r_{c}$ in the range $2 r_{c}<\delta$.

Based on these results, we can conclude that the curvature diameter and tip angle have qualitatively different influences on streaming vorticity, both inside and outside the VBL. Smaller and sharper structure provides stronger streaming force and flow.

\section{Streaming velocity magnitude per acoustic power}

We now aim to define a simple fitting parameter to quantify the efficiency of the response of streaming flow in regards to the prescribed vibration. The analyses in Ovchinnikov et al. [42] end up to a general expression for the streaming velocity in cylindrical coordinate $(r, \phi)$ as:

$$
v_{s}(r)=\frac{v_{a}^{2}}{v} \frac{\delta^{2 n-1}}{a^{2 n-2}} H_{\alpha}\left(\frac{r}{\delta}\right),
$$

where $n$ is a coefficient that depends on $\alpha, n=\frac{\pi}{2 \pi-\alpha} ; a$ is a length scale close to that of the sharp-edge height $h$. The function $H_{\alpha}\left(\frac{r}{\delta}\right)$ contains the radial profile of the streaming flow. Quantitatively, we mainly focus on the characteristic (and maximal) value of $v_{s}(r)$ at $r=\delta$ and $\phi=0$, so in what follows we shall just consider the constant prefactor $\frac{1}{v} \frac{\delta^{2 n-1}}{a^{2 n-2}}$ that relates $v_{s}$ to $v_{a}^{2}$. Let us note that this equation, supposedly valid in the range $r_{c}<\delta$, does not contain any dependence on $r_{c}$. Conversely, the theory by Doinikov et al. contains a dependence on $r_{c}$ [43]. 


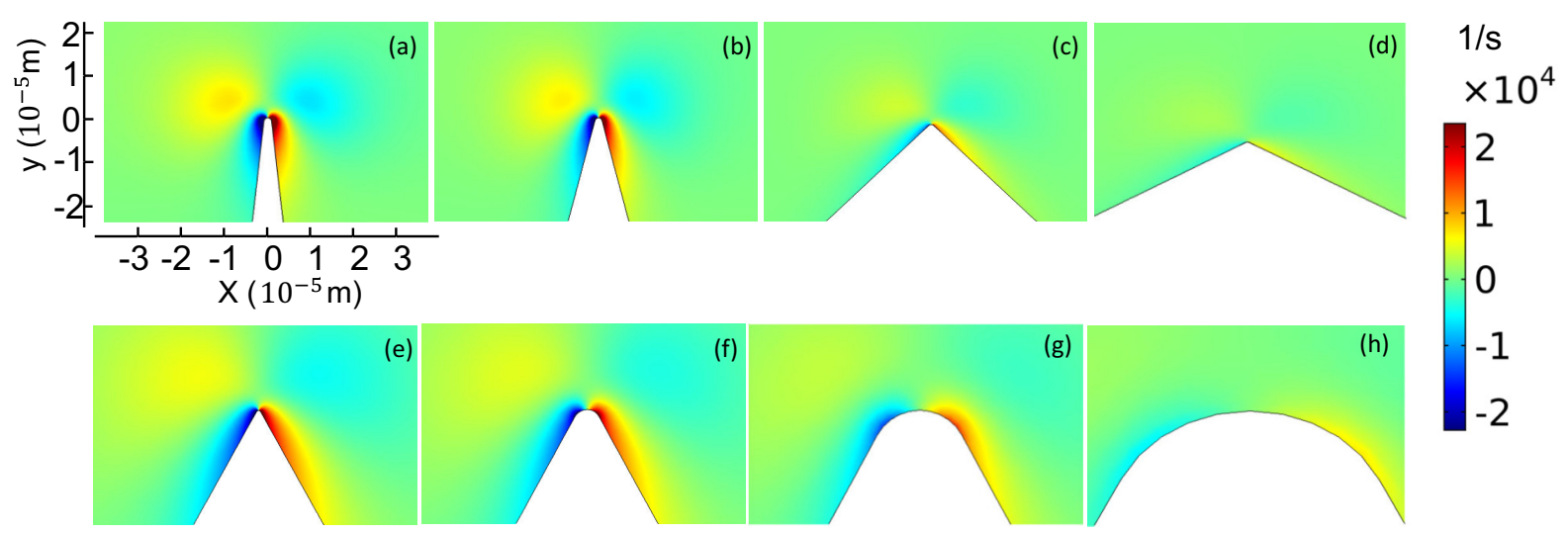

FIG. 6. The vorticity maps of the streaming flow in the region close to the tip are represented under different geometrical conditions. Red color (positive vorticity) and blue color (negative vorticity) respectively correspond to flows in counterclockwise and clockwise directions. For all maps, the vibration velocity $v_{a}=101.7 \mathrm{~mm} / \mathrm{s}, f=2500 \mathrm{~Hz}$, and liquid is water, so that $\delta \simeq 11.3 \mu \mathrm{m}$. Panels (a)-(d) correspond to the same curvature diameter $2 r_{c}=2.8 \mu \mathrm{m}$ but different tip angles $\alpha$ : (a) $\alpha=12^{\circ}$, (b) $\alpha=30^{\circ}$, (c) $\alpha=90^{\circ}$, (d) $\alpha=120^{\circ}$. Panels (e)-(h) correspond to the same tip angle $\left(\alpha=60^{\circ}\right)$ but different curvature diameters: (e) $2 r_{c}=1.0 \mu \mathrm{m}$, (f) $2 r_{c}=6 \mu \mathrm{m},(\mathrm{g}) 2 r_{c}=20 \mu \mathrm{m}$, (h) $2 r_{c}=50 \mu \mathrm{m}$.

The results presented in Fig. 5 confirm that for a given combination of geometry, acoustic frequency, and liquid viscosity - and actually for most experimental conditions $v_{\mathrm{sm}}$ - varies quadratically with the amplitude of vibration velocity $v_{a}$. Therefore, we define the fitting parameter $\theta=\frac{\Delta v_{\mathrm{m}}}{\Delta\left(v_{a}^{2}\right)}$ as a measurement of the efficiency of the momentum conversion from acoustic to streaming flows. In the following, we shall consider $\theta$ to quantify the influence of the different varying parameters, namely $\alpha, r_{c}$, and $\nu$. Similarly, we define $\theta^{\prime}=\frac{\Delta v_{s m}^{\prime}}{\Delta\left(v_{a}^{2}\right)}$.

\section{Influence of tip angle}

In this first series of results, we quantify the strength of the streaming flow for different values of angle $\alpha$, from $12^{\circ}$ to $180^{\circ}$, keeping all other quantities constant. In particular, as illustrated in the vorticity maps of Figs. 6(a)-6(d), $r_{c}$ can be kept constant for different $\alpha$, except of course for $\alpha=180^{\circ}$ that corresponds to case of a flat, straight wall. Figure 7 shows $v_{\mathrm{sm}}$ versus $v_{a}^{2}$ for different values of $\alpha$. As previously stated, the more acute the angle, the stronger the streaming flow for a given $v_{a}$. Besides, a flat wall with $\alpha=180^{\circ}$ does not generate any streaming flow even for high $v_{a}$.

Since the vast majority of cases exhibited a robust quadratic dependence between $v_{\mathrm{sm}}$ and $v_{a}$, we extracted $\theta$ for each value of $\alpha$. The results are shown in Fig. 8, where $\theta$ is plotted versus $(180-\alpha)$.

Keeping all other parameters constant, here $2 r_{c}=2.8 \mu \mathrm{m}$, $f=2500 \mathrm{~Hz}$ and liquid properties being those of water $(\delta$ $=11.5 \mu \mathrm{m}), \theta$ achieves its highest value with the sharpest angle, $\alpha=12^{\circ}$. The maximal efficiency of the momentum conversion is slightly below $10^{-2} \mathrm{~s} / \mathrm{mm}$. When $\alpha$ reaches $90^{\circ}, \theta$ drops to roughly $3 \times 10^{-3} \mathrm{~s} / \mathrm{mm}$, and it vanishes to zero when $\alpha$ approaches $180^{\circ}$. Thus the dependence of $\theta$ with $\pi-\alpha$ is strongly nonlinear.

\section{Influence of tip curvature}

We now investigate the influence of $r_{c}$ on $\theta$, for a series of four values of $\alpha$, from $12^{\circ}$ to $120^{\circ}$. As previously, simulations were carried out under the same liquid viscosity (water, $v=$
$10^{-6} \mathrm{~m}^{2} / \mathrm{s}$ ) and frequency $f=2500 \mathrm{~Hz}$, so that $\delta$ was kept constant at $11.5 \mu \mathrm{m}$ and only $r_{c}$ was varied. Figure 9 shows the dependence of $\theta$ versus $2 r_{c} / \delta$.

These results reveal a decrease of $\theta$ with $r_{c}$, and this decrease becomes more significant within the range $2 r_{c}>\delta$, see Fig. 9. Let us note that in Fig. 9, we also put results from simulations using the PT method for the two extreme values of $\alpha$, again in the aim to illustrate the gap between both methods. This confirms that PT systematically overestimates the magnitude of the streaming flow by a factor of roughly 1.2 .

This constitutes a quantitative confirmation of what was suggested in the vorticity maps of Figs. 6(a)-6(d). Also, the influence of $r_{c}$ is more pronounced when the tip angle is more acute.

Once $2 r_{c}$ is increased and become larger than $\delta, \theta$ significantly decreases, which is observed for all tip angles

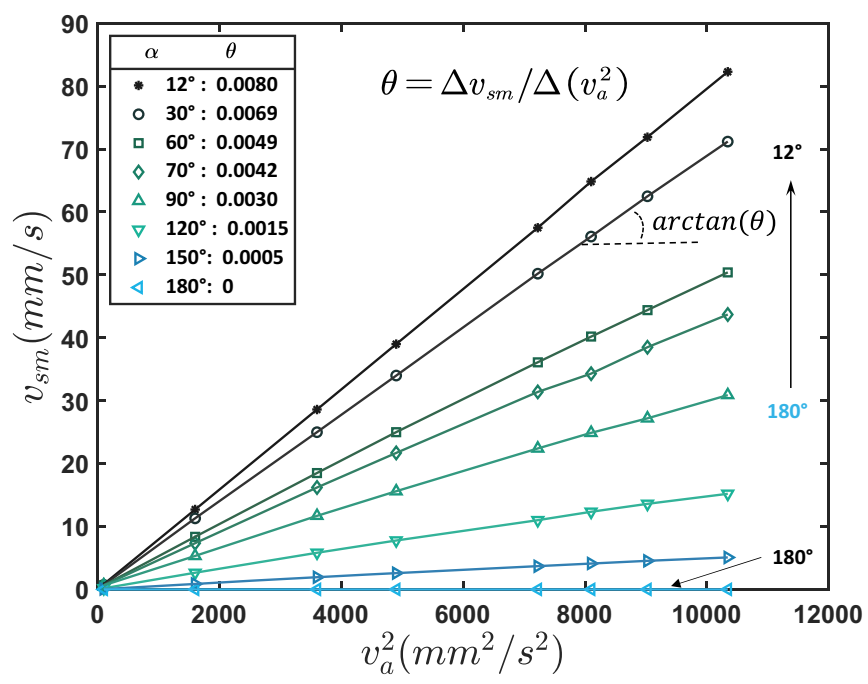

FIG. 7. The maximum velocity is plotted versus the square of the vibration velocity $v_{a}^{2}$, with different tip angles. The coefficient $\theta$ is extracted from a linear fit, which holds very well within the whole range of $v_{a}$. Other conditions are $2 r_{c}=2.8 \mu \mathrm{m}$ and $f=2500 \mathrm{~Hz}$. 


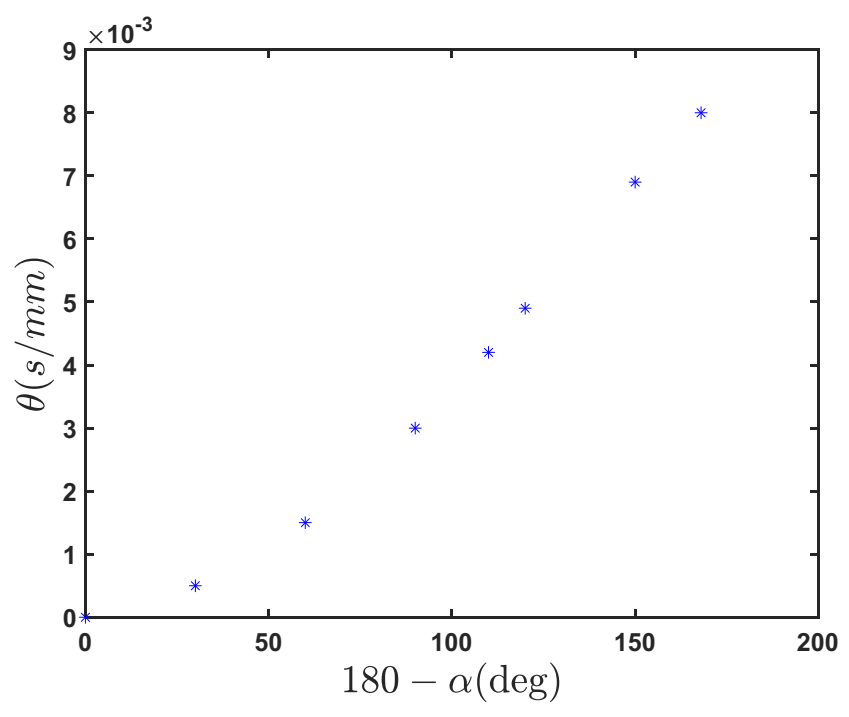

FIG. 8. The coefficient $\theta=\Delta v_{\mathrm{sm}} / \Delta\left(v_{a}^{2}\right)$ (efficiency of the momentum conversion from acoustic to streaming flows) is plotted versus the supplementary of the tip angle $180-\alpha$. Other conditions are $2 r_{c}=2.8 \mu \mathrm{m}$ (except for $\alpha=180^{\circ}$ where $r_{c}$ is infinite) and $f=2500 \mathrm{~Hz}$.

(Fig. 9). This is in accordance with the spreading and weakening contour observed in Figs. 6(g) and 6(h). Hence in the range $2 r_{c}>\delta$, the conversion of acoustic power into streaming flow is less efficient, which again emphasizes that the sharpness of the structure is determinant for the generation of intense streaming. In other words, when the tip is no longer sharp, the magnitude of AS weakens as we should retrieve the classical Rayleigh-Schlichting streaming. Let us mention that the analytical prediction of Doinikov et al.'s

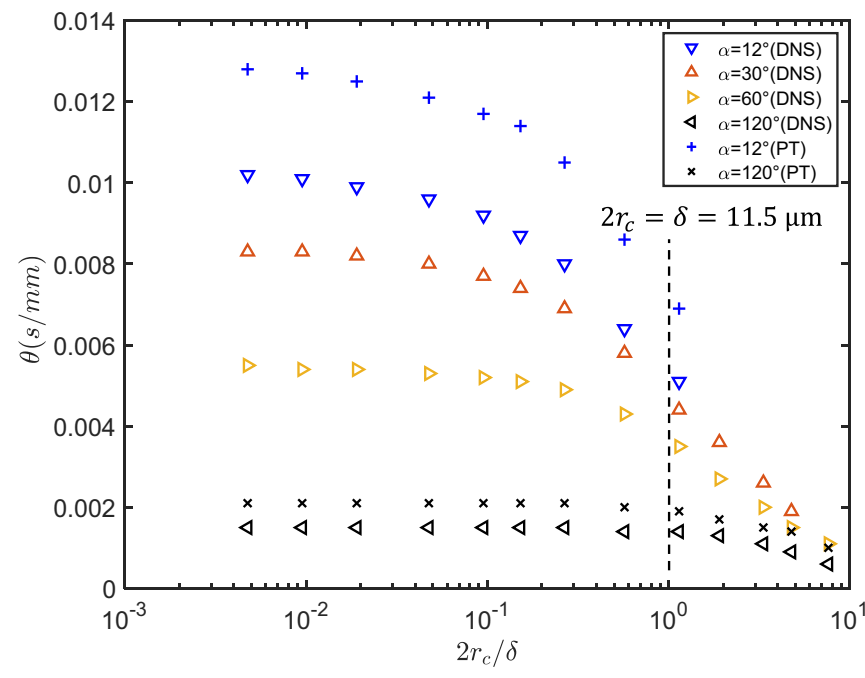

FIG. 9. The coefficient $\theta=\Delta v_{\mathrm{sm}} / \Delta\left(v_{a}^{2}\right)$ (efficiency of momentum conversion from acoustic to streaming flows) based on the maximal value of streaming velocity, is plotted versus ratio between curvature diameter $2 r_{c}$ and boundary layer thickness $\delta$ for four different tip angles $\alpha$. DNS results should be considered as reliable and PT simulation appears to overestimate the result according to the two extreme cases $\left(\alpha=12^{\circ}\right.$ and $\left.\alpha=120^{\circ}\right)$.

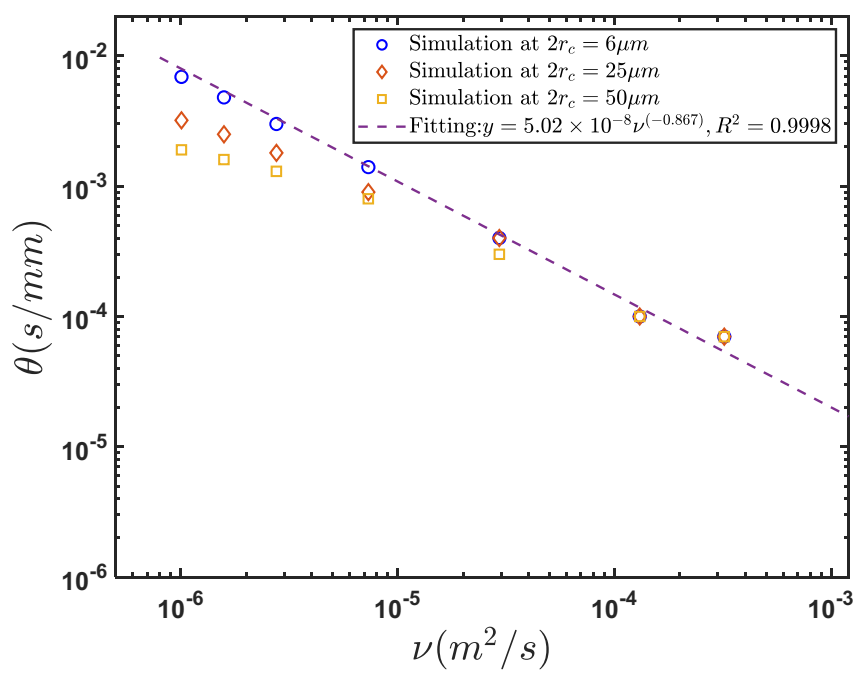

FIG. 10. The coefficient $\theta=\Delta v_{\mathrm{sm}} / \Delta\left(v_{a}^{2}\right)$ (efficiency of momentum conversion from acoustic to streaming flows) is plotted versus kinematic viscosity $\nu$. Other parameters are $\alpha=30^{\circ}$ and $f=2500 \mathrm{~Hz}$. The fitting power-law curve is based on the results for $2 r_{c}=6 \mu \mathrm{m}$. For $v>5 \times 10^{-5} \mathrm{~m}^{2} / \mathrm{s}$, the data points coincide with each others, showing that $\theta$ is almost independent on $r_{c}$.

study (Eq. (27) of Ref. [43]) suggests a power-law dependence on $r_{c}$ of the streaming velocity. However, it is hard to directly compare it with our results, since the values of $r_{c} / \delta$ in this latter study can be very different from those used here.

\section{Influence of viscosity}

One of the remarkable and nonintuitive features of Rayleigh-Schlichting streaming is its independence on viscosity, providing that the typical size of the container is much larger than the thickness of the VBL, $\delta[14,20]$. This classical result, which expresses that streaming is both spawned and hindered by viscosity, can be retrieved by simple scaling arguments [48,51], though it is no longer true in confined geometries [51]. Here in the case of sharp-edge streaming, we show that, despite that $\delta$ can remain small compared to the channel size, viscosity has a strong influence on the sharpedge-induced streaming. As previously, we extracted $\theta$ as a fitting parameter that relates $v_{\mathrm{sm}}$ with $v_{a}^{2}$ over a large range of $v_{a}$ for different values of $v$ and keeping $r_{c}$ and $\alpha$ at fixed values.

Figure 10 shows a strong decrease of $\theta$ with kinematic viscosity $v$ in Log-Log axes. We span a large range of values for $v$, from that of water $\left(10^{-6} \mathrm{~m}^{2} / \mathrm{s}\right)$ to a $1000 \times$ more viscous liquid, with a corresponding $\delta \simeq 357 \mu \mathrm{m}$, which in practice would correspond for instance to pure glycerin. In particular for $2 r_{c}=6 \mu \mathrm{m}$, together with $f=2500 \mathrm{~Hz}$ and constant $\alpha=30^{\circ}$, we remain in a sharp-edge streaming situation since $2 r_{c}<\delta$. The decrease can be well fitted by a power law, with an exponent of -0.867 giving the best fit, see Fig. 10 .

We now change the value of $2 r_{c}$ from $6 \mu \mathrm{m}$ to 25 and $50 \mu \mathrm{m}$. The cases investigated with $2 r_{c}=25$ and $50 \mu \mathrm{m}$ reveal that the decrease of $\theta$ with viscosity is much less pronounced for higher values of $2 r_{c} / \delta$, hence in the lower 
viscosity range. Actually, for these two values, the evolution of $\theta$ shows a crossover from sharp-edge streaming to classical Rayleigh-Schlichting streaming. Therefore if $2 r_{c}>\delta$, it turns out that the dependence of $\theta$ on viscosity is not captured by a power law. Let us note that, while we ran our simulations up to $v=10^{-3} \mathrm{~m}^{2} / \mathrm{s}$, the relationship between $v_{\mathrm{sm}}$ and $v_{a}^{2}$ is no longer purely linear within this high-viscosity range. Therefore, the value of $\theta$ could not be extracted for the highest values of $v$.

Conversely, the value of $\theta$ is independent on $r_{c}$ in the highviscosity range, i.e., when $2 r_{c} / \delta<1$ : This is a trademark of sharp-edge streaming.

Equation (10) taken from Ovchinnikov et al.'s study [42] predicts a decrease of $\theta$ with $v$ via a power law of negative exponent, as $\theta \sim v^{\left(n-\frac{3}{2}\right)}$. For the chosen angle $\theta=30^{\circ}, n \simeq$ 0.54 , yielding an exponent $\left(n-\frac{3}{2}\right)$ of -0.96 , close to but different from the value of -0.867 found empirically. The corresponding analytical predictions of Doinikov et al.'s study [43] propose an exponent of -1 .

Let us here give more details on an apparent contradiction between the results of Fig. 9 and those of Fig. 10, concerning the dependence of $\theta$ with $v$. The results of Fig. 9 were obtained by varying $r_{c}$ while keeping $\nu, \omega$ constant (and thus $\delta$ at $11.5 \mu \mathrm{m}$ ). Conversely, the results of Fig. 10 were obtained by varying $v$, while keeping $r_{c}$ constant for the same set of data. Hence, the apparent paradox of the influence of $v$ on $\theta$ comes from the following:

(i) The decrease of $\theta$ with $r_{c} / \delta$ in Fig. 9 is attributed to that the streaming is weaker when $r_{c}$ increases, in particular when it becomes of the same order as, or larger than, $\delta$ : In this latter condition, the tip is no longer considered as sharp with respect to $\delta$. Conversely in a situation of sharp edge streaming, the streaming flow is almost independent on $r_{c}$, as shown in the left region of the plot.

(ii) The decrease of $\theta$ with $v$ is effective only in the situation of sharp edge streaming, where $r_{c}$ is significantly smaller than $\delta$. If this latter condition is true for water, then it will of course remain true for more viscous liquids, as for the results in Fig. 10.

The dependence of $\theta^{\prime}$ with $v$ also shows a global decrease, see Fig. 11. But the main difference with $\theta$ is that within the range where $r_{c} / \delta$ is large enough, hence, the edge is not sharp, the value of $\theta^{\prime}$ is independent on $v$. It is clearly evidenced for $r_{c}=50 \mu \mathrm{m}$ in the low-viscosity range. The distinction between $\theta$ and $\theta^{\prime}$ is mostly significant where $r_{c} / \delta>$ 1 , which corresponds to situations depicted in the vorticity maps of Figs. 6(g) and 6(h). In these situations, the maximum of streaming velocity is not localized along the $y$ axis and ressembles classical Rayleigh streaming. In any case, the behavior of $v_{\mathrm{sm}}^{\prime}$ follows a quadratic increase with $v_{a}$, so that $\theta^{\prime}$ remains well defined. More surprisingly, beyond $v=10^{-4}$ $\mathrm{m}^{2} / \mathrm{s}$, the decrease of $\theta^{\prime}$ with $v$ deviates from a power law. Also $\theta^{\prime}$ remains dependent on $r_{c}$ in the whole range of $v$ investigated.

\section{DISCUSSIONS AND CONCLUSION}

Let us now recall and summarize the main results. Motivated by experimental results on the generation of intense acoustic streaming near sharp edges [33-37,42,45], the re-

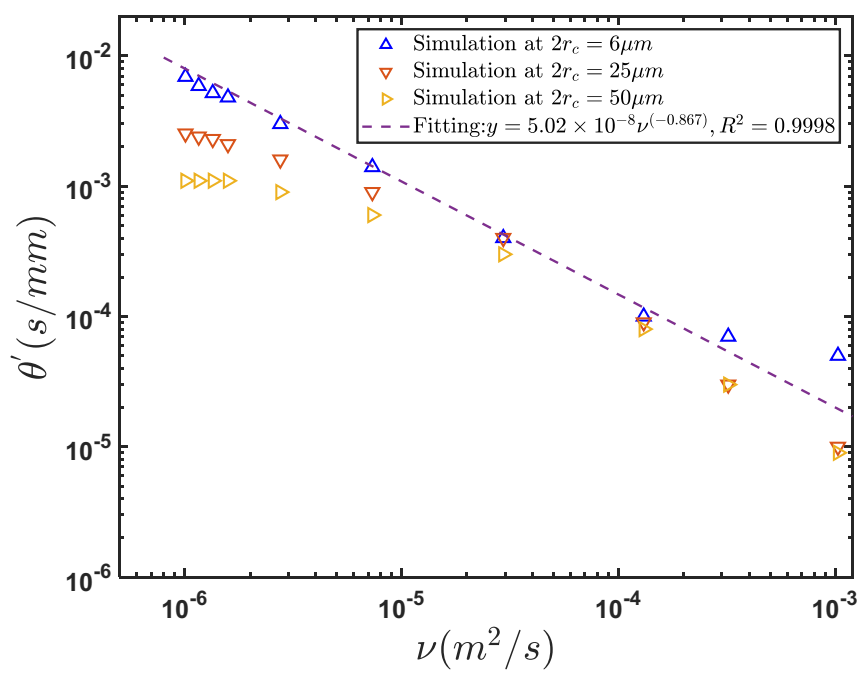

FIG. 11. The coefficient $\theta^{\prime}$ is plotted versus kinematic viscosity v. $\theta^{\prime}=\Delta v_{\mathrm{sm}}^{\prime} / \Delta\left(v_{a}^{2}\right)$, fitting efficiency from of the momentum conversion from acoustic to streaming flows. Other parameters are $\alpha$ $=30^{\circ}, f=2500 \mathrm{~Hz}$. The fitting power-law curve is based on the simulation when $2 r_{c}=6 \mu \mathrm{m}$.

sults of our DNS simulations allow a characterization of the streaming flow both outside and inside the VBL, here of typical thickness $\delta=11.5 \mu \mathrm{m}$ for water at $f=2500 \mathrm{~Hz}$. This constitutes a significant step forward with respect to the state of the art, since it is experimentally hard to access the flow details within the VBL [37]. Furthermore, few studies employed the DNS method so far [43], and our study provided results of better precision than the classical PT, especially at high forcing. In particular, providing $2 r_{c}$ is smaller than $\delta$ (which is the case of sharp-edge streaming), the maximum of streaming velocity is found near the apex of the sharp tip, at a distance of roughly $y=\delta$, inducing regions of strong and concentrated vorticity aside and within the VBL, as well as larger outer vortices, which ensures efficient mixing across the whole channel [33,35-37].

Furthermore, we gained better understanding of the firstorder acoustic velocity field. It turns out that both the orientation of the oscillations and their amplitude are influenced by the sharp structure, which leads to a strong and sharply localized perturbation to the fluid. Namely, the norm of $\mathbf{v}_{a}(x, y)$ is maximal near the tip, precisely around the location of the maximum of streaming velocity $(x=0, y=\delta)$. Surprisingly, this velocity $v_{a}$ is found twice to three times larger than that far away from the tip. Let us note that this confirms recent experiments [37]. This effect, which significantly contributes to the streaming efficiency, depends on the sharp edge structure. Though, it remains to be explained and quantified in more details.

Our study also focuses on the influence of the tip sharpness and reveals that the two parameters $r_{c}$ and $\alpha$ are crucial for the generation of acoustic streaming. While their respective influences were difficult to dismantle in experiments, our numerical results provided a better understanding. Since the acoustic flow direction (angle $\alpha_{v b}$ ) follows that of the walls, the sudden change of oscillation orientation beside the tip leads to such a centrifugal effect. Along a typical length as 

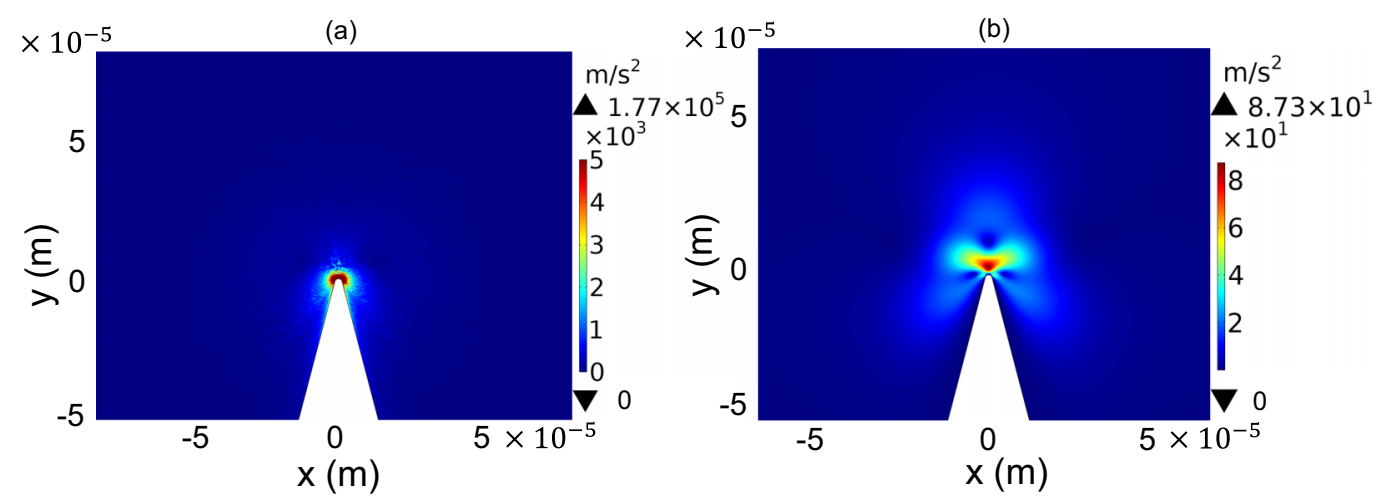

FIG. 12. The intensity map of quantities $\left\|\nu \nabla^{2} \mathbf{v}_{s}\right\|$ (a) and $\left\|\left(\mathbf{v}_{s} \cdot \nabla\right) \mathbf{v}_{s}\right\|$ (b) are shown for $v_{a}=70.5 \mathrm{~mm} / \mathrm{s}$. The left term has a maximum of $1.77 \times 10^{5} \mathrm{~m} / \mathrm{s}^{2}$, which is roughly 2000 times stronger than the right one $\left(87.3 \mathrm{~m} / \mathrm{s}^{2}\right)$. Close to the tip, the ratio is about 70 . $\left(\mathbf{v}_{s} \cdot \nabla\right) \mathbf{v}_{s}$ can thus be considered as negligible in Eq. (7).

short as $2 r_{c}$, the orientation jumps from $\alpha_{v b}=\pi / 2-\alpha / 2$ for $x \ll r_{c}$ to $\alpha_{v b}=\alpha / 2-\pi / 2$ for $x \gg r_{c}$, hence an overall rotation of $\Delta \alpha_{v b}=\pi-\alpha$. This gradient generates strong values for the effective streaming force $F_{s}$.

One of the remaining challenging issues, is the detailed determination of the radial and azimuthal flow profile. In this aspect, the study by Doinikov et al. constitutes a step forward and offers results complementary to ours. This latter study revealed in particular the occurrence of secondary vortices along the sharp edge, when the angle of the wedge $\alpha$ is large enough. The origin of these secondary vortices remain unclear and could be specific to situations of higher frequencies. Very recent experiments [45] also emphasized the nontrivial influence of $f$ and $v$ when $\delta$ is no longer small compared to channel depth and width.

Let us briefly comment on Eq. (8). Due to relatively local strong values for $\mathbf{v}_{s}$, i.e., comparable in magnitude to $\mathbf{v}_{a}$, the nonlinear term $\left(\mathbf{v}_{s} \cdot \nabla\right) \mathbf{v}_{s}$ should in principle be significant. The physical meaning of this term can be viewed as the self-advection of the streaming flow, which in practice leads to vortex elongation in Rayleigh-Schlichting streaming [52]. However, this is somewhat contradictory with the robust quadratic relationship found between $v_{\text {sm }}$ and $v_{a}$, regarding Eq. (8). To explain this apparent contradiction, we retain two possible hypotheses:

(i) Although the magnitude of $\mathbf{v}_{s}$ can be relatively large locally, the term $\left(\mathbf{v}_{s} \cdot \nabla\right) \mathbf{v}_{s}$ could be negligible, especially in the region around the maximum $v_{\mathrm{sm}}$.

(ii) The term $\left(\mathbf{v}_{s} \cdot \nabla\right) \mathbf{v}_{s}$ could be irrotational, so that it can be exactly compensated by the pressure gradient term $\nabla p_{s}$.

To check these two assumptions, we plotted the maps of the norms of both quantities $\left(\mathbf{v}_{s} \cdot \nabla\right) \mathbf{v}_{s}$ and $\nu \nabla^{2} \mathbf{v}_{s}$. The results are shown in Fig. 12 for a typical value of $v_{a}$ in the intermediate range. It turns out that the first assumption is the right one, as it shows that the magnitude of $\left\|\nu \nabla^{2} \mathbf{v}_{s}\right\|$ overcomes that of $\left\|\left(\mathbf{v}_{s} \cdot \nabla\right) \mathbf{v}_{s}\right\|$ by a factor of roughly 70 . Therefore, the nonlinear term $\left(\mathbf{v}_{S} \cdot \nabla\right) \mathbf{v}_{s}$ can be considered as negligible in Eq. (8), which explains the extension of the quadratic behavior between $v_{\mathrm{sm}}$ and $v_{a}$ in sharp-edge streaming. Furthermore, it underlines that the differences between PT and DNS simulation results, and the fact that DNS better matches experiments of sharp-edge streaming should be explained by the impor- tance of the other nonlinear terms $\left(\mathbf{v}_{a} \cdot \nabla\right) \mathbf{v}_{s}$ and $\left(\mathbf{v}_{s} \cdot \nabla\right) \mathbf{v}_{a}$ in Eq. (6).

In the seek for optimal operating conditions of sharp edge AS, the efficiency of conversion from acoustic vibrations to streaming flow is quantified by $\theta$. In particular, while the fabrication of sharp tips requires in practice careful and expensive techniques, especially for $r_{c}$ as small as a few microns, Fig. 9 shows that the streaming flow does not gain much in strength when $r_{c}$ is lowered below $\delta / 2$. The precise identification of the influence of $r_{c}$ and $\alpha$ was made possible thanks to the DNS simulations.

The role of viscosity was also investigated. The power-law decrease of $\theta$ with $v$, predicted by Ovchinnikov et al. [42] was confirmed by our simulations, although the exponent was found weaker than the predicted one. We also confirmed the independence of $\theta$ on $r_{c}$ in the range $2 r_{c}<\delta$, and we investigated the crossover between the sharp edge AS and classical Rayleigh streaming regimes by tuning the value of $2 r_{c} / \delta$. In particular, we recover the independence of $\theta$ on $v$ if $2 r_{c} / \delta \gg 1$ (as in classical Rayleigh streaming).

Also, our simulations showed that the quadratic relationship $v_{\mathrm{sm}} \sim v_{a}^{2}$ fails at high-enough viscosity. This has to be considered as a geometrical constraint, since with higher range of $v_{a}$, the size of the outer vortices is comparable to that of the channel width $w$. For high-enough $v_{a}$, the streaming can then be limited by the size of the microchannel.

Let us also suggest a quantitative criterion of efficiency in the context of (macro-)mixing under a typical imposed flowrate $Q$ through the channel. Previous experiments quantifying both the maximal streaming velocity and mixing efficiency revealed that a satisfying mixing rate could be obtained if the averaged flow velocity, here $\langle V\rangle=\frac{Q}{w d}$, was comparable to the maximal streaming velocity [37]. For the width $w=500 \mu \mathrm{m}$ and depth $d=50 \mu \mathrm{m}$ used in these experiments, and a middle-range value of $Q=10 \mu \mathrm{l} / \mathrm{mn}$, it yields: $\langle V\rangle \simeq 6.7 \mathrm{~mm} / \mathrm{s}$. Therefore in practice, the velocity set point $v_{\mathrm{sm}}=\langle V\rangle$ shall be related to specific conditions on both the tip geometry and liquid viscosity, both ruling the value of $\theta$, also to take into account the maximal $v_{a}$ that the transducer can generate. It is worth noting though, that micromixing at the molecular scale also depends on the form of vortices generated by AS and one of our upcoming study 
addresses this issue using iodate-iodide reaction as a chemical probe $[53,54]$.

To sum up, the FEM-based DNS method gives very satisfactory agreement with experimental results and it overperforms the classical PT model. The latter does not consider the nonlinear terms in the streaming force calculation and tends to overestimate the streaming velocity. In this sense, our study shows that, providing the right boundary conditions are prescribed and all nonlinear terms are kept in the calculation, AS streaming can be successfully studied in a quantitative way, with minimal inexpensive computing material, i.e., without computer cluster nor MP and FEM commercial software. In this sense, we can finally state that the assumption of a 2D flow is necessary to carry out DNS simulations by keeping a reasonable computational cost, which would be very expensive for simulations under an equivalent 3D geometry. Still, we checked that the flow remained $2 \mathrm{D}$, even by prescribing a 3D geometry similar to that of the experimentally studied channel, under a few typical situations. This is presumably due to the high width:depth ratio in our channel geometry.

\section{ACKNOWLEDGMENT}

C.Z. was funded by the China Scholarship Council.

\section{APPENDIX}

\section{Perturbation theory and its implementation}

The perturbation theory is generally well adapted to address acoustofluidics problems in the framework of "weak disturbances." With limited access to computational fluid dynamics (CFD), PT is a very powerful tool to reduce the N-S equation, which potentially includes nonlinear terms that couple the acoustic and streaming velocity fields, into a simpler one. Therefore, PT provides an convenient method to bring out the physical fundamental core of the acoustic streaming problems while retaining relatively simple mathematical formulation [15,16,21,42,46,47].

For the present study, $v_{a}$ and $v_{s}$ are governed by both Eqs. (6) and (7), which set respectively the oscillating and steady terms in the velocity field. The PT assumes $v_{a} \gg v_{s}$ so that the inertial terms in the Eq. (6), $\left(\mathbf{v}_{s} \cdot \nabla\right) \mathbf{v}_{a}$ and $\left(\mathbf{v}_{a} \cdot \nabla\right) \mathbf{v}_{s}$, can be neglected. Without these terms, Eqs. (6) and (7) can then be solved separately to obtain $v_{a}$ and $v_{s}$.

The procedure of the calculation based on PT can be proceeded following two steps: (i) solving the wave equation Eq. (6) to determine the vibration velocity field in the geometry structure, with first-order time-periodic terms, and (ii) solving the streaming equation Eq. (7), in which the force term in Eq. (9) can be determined by the results of the previous step. The second-order terms are steady ones, from which the streaming velocity $v_{s}$ is deduced.

Although Ovchinnikov et al. [42] pointed out the limitation of PT with respect to DNS method, PT remains a powerful framework to analyze the underlying physics of the streaming fields near the sharp tip, especially when the vibration amplitude within the liquid is small enough so that the acoustic Reynolds number $\operatorname{Re}_{a}=\frac{A \omega h}{v}$ remains of the order of one or lower.

In COMSOL, basic steps to implement perturbation theory are as follows: (i) Module "Thermoviscous Acoustics, Frequency Domain" for solving the acoustic vibration velocity field; the following set of equations are computed:

$$
\begin{gathered}
i \omega \rho_{a}=-\nabla \cdot\left(\rho_{0} \mathbf{v}_{a}\right), \\
i \omega \rho_{0} \mathbf{v}_{a}=\nabla \cdot\left\{-p_{a} \mathbf{I}+\mu\left[\nabla \mathbf{v}_{a}+\left(\nabla \mathbf{v}_{a}\right)^{T}\right]\right. \\
\left.-\left(\frac{2}{3} \mu-\mu_{B}\right)\left(\nabla \cdot \mathbf{v}_{a}\right) \mathbf{I}\right\} \\
\rho_{a}=\rho_{0}\left(\beta_{T} p_{a}-\alpha_{P} T_{a}\right),
\end{gathered}
$$

where the subscript $\omega$ denotes the complex, time-periodic component of any quantity, $\beta_{T}$ is the isothermal compressibility coefficient, $\alpha_{0}$ is the coefficient of thermal expansion, $\mu$ and $\mu_{B}$ are respectively the shear and bulk dynamic viscosities. I stands for the identity matrix. Let us remark that although for the sake of rigour we chose to keep terms for compressible and thermal effects, we checked that these effects were negligible in the whole range of the simulations.

(ii) Module "Laminar Flow" for solving the streaming velocity field,

$$
\begin{gathered}
\rho\left(\mathbf{v}_{s} \cdot \nabla\right) \mathbf{v}_{s}=\nabla \cdot\left[-p_{s} \mathbf{I}+\mathbf{K}\right]+\mathbf{F}, \\
\mathbf{K}=\mu\left[\nabla \mathbf{v}_{s}+\left(\nabla \mathbf{v}_{s}\right)^{T}\right], \\
\rho \nabla \cdot \mathbf{v}_{s}=0, \\
\mathbf{F}_{s}=\left\langle\rho_{a} \frac{\partial \mathbf{v}}{\partial t}\right\rangle+\rho_{0}\langle(\mathbf{v} \cdot \nabla) \mathbf{v}\rangle,
\end{gathered}
$$

with $\mathbf{F}_{s}$ being the "volume force" inserted into the model, computed from the time-average over one period. $\mathbf{K}$ stands for the viscous shear stress matrix associated to the streaming flow.

(iii) Boundary conditions: To solve the vibration velocity, the left and right boundaries (labelled as 1 and 6) are set respectively (left, 1) with the acoustic velocity oscillating at the prescribed value of amplitude in the normal direction and (right, 6) with the pressure at $p_{0}$. Other boundaries are set as no-slip walls.

For the second-order streaming velocity, the left and right sides of the domain are set as inlet and outlet at given incoming velocity, here taken equal to zero. The pressure at the outlet is set constant at $p_{0}$. The other boundaries are set to be no-slip walls.

\section{Direct numerical simulation implementation}

The detailed description of DNS has been given in Sec. II. Implementing DNS in COMSOL includes the following steps:

(i) Module "Laminar Flow" for direct solving the NavierStokes equation with periodic velocity boundary conditions,

$$
\begin{gathered}
\rho \mathbf{\nabla} \cdot \mathbf{v}=0 \\
\rho \frac{\partial \mathbf{v}}{\partial t}+\rho(\mathbf{v} \cdot \nabla) \mathbf{v}=\nabla \cdot[-p \mathbf{I}+\mathbf{K}], \\
\mathbf{K}=\mu\left[\nabla \mathbf{v}+(\nabla \mathbf{v})^{T}\right] .
\end{gathered}
$$

Here the effective force generating the streaming flow does not appear explicitly but is imbedded in the nonlinear term. 

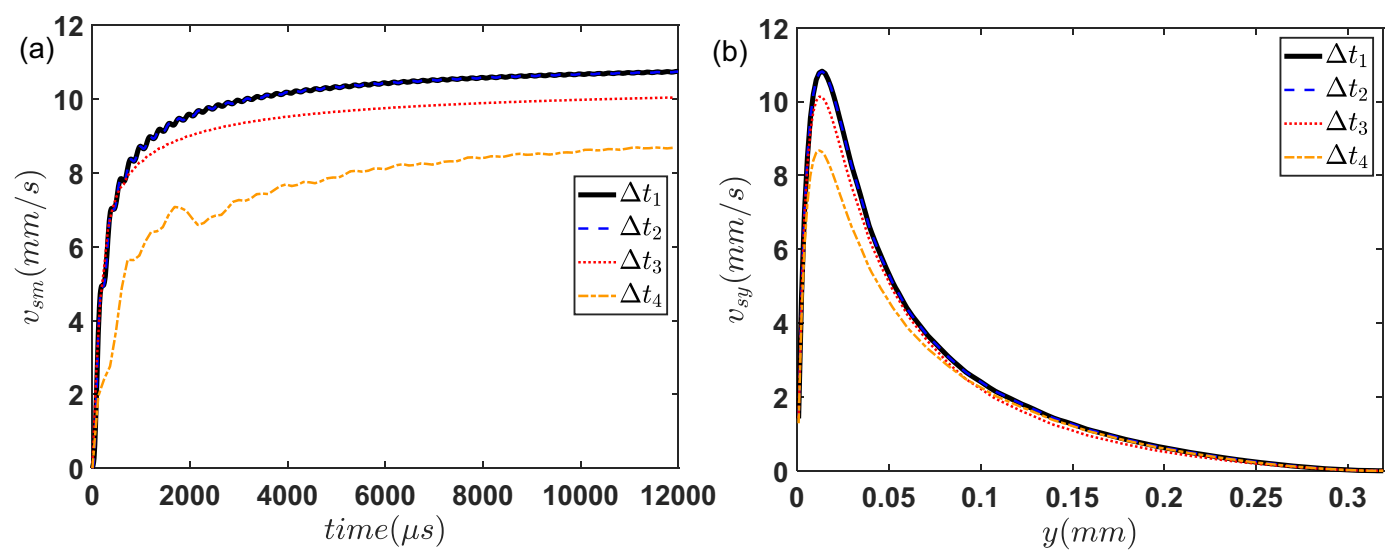

FIG. 13. The variation of streaming velocity is represented versus numerical iteration time (a), and the steady $y$ direction streaming velocity is shown at different time steps (b). Time steps $\Delta t_{1}=1 \mu \mathrm{s}, \Delta t_{2}=8 \mu \mathrm{s}, \Delta t_{3}=80 \mu \mathrm{s}, \Delta t_{4}=120 \mu \mathrm{s}$ correspond to $1 / 400$ th, $1 / 50$ th, $1 / 5$ th, and $1 / 3.33$ rd of an acoustic period. The whole duration of the simulation equals 30 acoustic periods.

(ii) Module "Domain ODEs and DAEs" for calculating the time average values of the velocity field in step 1 . The streaming flow is here computed via a numerical integration:

$$
\mathbf{v}_{s}=\frac{1}{\Delta t} \int_{t}^{t+\Delta t} \mathbf{v} d t,
$$

with $\Delta t=\frac{n}{f}, n$ being an integer representing the number of periods for the time-averaging.

(iii) Boundary conditions: The acoustic velocity (in form of a sinusoidal function of time) is set as the left boundary condition and the right boundary condition is set as a constant pressure $p_{0}$. Other boundaries are set as no-slip walls.

\section{Mesh and grid independence study}

The mesh grid is built with triangle elements, with the maximum element size being $0.014 \mathrm{~mm}$, and the minimum one being $0.0002 \mathrm{~mm}$. Smooth transition is performed with a maximum element growth rate of 1.1. Close to the sharp edge, the mesh is refined by inflation layers to better account for the strong velocity gradients inside the VBL. The number of the layers is 3 and the layer stretching factor is 1.2 .

The mesh independence is assessed by comparing the results from the chosen mesh with those obtained in a refined mesh, which is generated by increasing the number of cells by $30 \%$. Comparing the two meshes, the obtained streaming velocity value differs by less than $1 \%$. The current mesh is thus considered as being as satisfactory balance between both in terms of accuracy, reliability, and computing time.

\section{Time to reach steady streaming field and time step}

For the PT method, the two-steps procedure consists in a readily computation process. For the DNS, however, the streaming flow appears after a transient state and thus needs some time to be fully developed and reach its steady state. As shown in Fig. 13(a), the streaming velocity $v_{\text {sm }}$ (the time average of the total velocity from the beginning of the simulation to a given time) grows with the number time steps until reaching a steady state. The corresponding time duration is roughly $12 \mathrm{~ms}$ and hence 30 acoustic cycles under the acoustic frequency of $2500 \mathrm{~Hz}$ (period of $400 \mu \mathrm{s}$ ).

The value of the time step is also essential to meet the Courant-Friedrichs-Lewy (CFL) stability condition. The Courant number, given by CFL $=v_{a} \Delta t / \Delta x$, should be kept lower than 1 to guarantee the numerical iteration stable [55]. As shown in Fig. 13, we test four time steps from 1 to 120 $\mu \mathrm{s}$ or from $1 / 400$ th to $3 / 10$ th of an acoustic period. Only $\Delta t_{4}=120 \mu \mathrm{s}$ gives a CFL higher than unit but $\Delta t_{3}=80 \mu \mathrm{s}$ is not fine enough to give a satisfactory maximum streaming velocity $v_{\text {sm }}$, see Fig. 13(a) and a reliable streaming distribution along the $y$ direction $v_{\mathrm{sy}}(y)$, see Fig. 13(b). We thus choose $\Delta t_{2}=8 \mu \mathrm{s}$ as a compromise since it gives the same results as $\Delta t_{1}=1 \mu \mathrm{s}$ but with a shorter computing time.

With the chosen time step of $8 \mu \mathrm{s}$ and a total of 30 acoustic cycles, the DNS computing duration is about $25 \mathrm{mn}$ per case study on an Intel i5-7500 CPU and 16 GB of RAM.

\section{Convective versus viscous terms}

Equation (7) suggests that the quadratic dependence of $v_{\mathrm{sm}}$ with $v_{a}$ should be right only if the term $\left(\mathbf{v}_{s} \cdot \nabla\right) \mathbf{v}_{s}$ is negligible compared to the other ones. Therefore, we compared the relative magnitude of $\left\|\nu \nabla^{2} \mathbf{v}_{s}\right\|$ and $\left\|\left(\mathbf{v}_{s} \cdot \nabla\right) \mathbf{v}_{s}\right\|$ in the form of colormaps shown in Fig. 12. The chosen $v_{a}=70.5 \mathrm{~mm} / \mathrm{s} \mathrm{cor-}$ responds to a value in the median range of investigation, but this remains true even for the largest investigated $v_{a}$, i.e., 107 $\mathrm{mm} / \mathrm{s}$. This confirms that although $\mathbf{v}_{s}$ can be comparable to $\mathbf{v}_{a}$ in magnitude, the term $\left(\mathbf{v}_{s} \cdot \nabla\right) \mathbf{v}_{s}$ remains small compared to the others of Eq. (7).
[1] M. Faraday, Philos. Trans. R. Soc. London 121, 299 (1831).

[2] J. Friend and L. Y. Yeo, Rev. Mod. Phys. 83, 647 (2011).

[3] K. Sritharan, C. J. Strobl, M. F. Schneider, and A. Wixforth, Appl. Phys. Lett. 88, 054102 (2006).
[4] T. Franke, S. Braunmuller, L. Schmid, A. Wixforth, and D. A. Weitz, Lab Chip 10, 789 (2010).

[5] A. Lenshof, C. Magnusson, and T. Laurell, Lab Chip 12, 1210 (2012). 
[6] S. S. Sadhal, Lab Chip 12, 2600 (2012).

[7] P. B. Muller, M. Rossi, Á. G. Marín, R. Barnkob, P. Augustsson, T. Laurell, C. J. Kähler, and H. Bruus, Phys. Rev. E 88, 023006 (2013).

[8] N. R. Skov, P. Sehgal, B. J. Kirby, and H. Bruus, Phys. Rev. Appl. 12, 044028 (2019).

[9] W. Qiu, J. T. Karlsen, H. Bruus, and P. Augustsson, Phys. Rev. Appl. 11, 024018 (2019).

[10] G. A. Voth, B. Bigger, M. R. Buckley, W. Losert, M. P. Brenner, H. A. Stone, and J. P. Gollub, Phys. Rev. Lett. 88, 234301 (2002).

[11] G. Vuillermet, P.-Y. Gires, F. Casset, and C. Poulain, Phys. Rev. Lett. 116, 184501 (2016).

[12] M. Legay, B. Simony, P. Boldo, N. Gondrexon, S. Le Person, and A. Bontemps, Ultrason. Sonochem. 19, 1194 (2012).

[13] B. G. Loh, S. Hyun, P. I. Ro, and C. Kleinstreuer, J. Acoust. Soc. Am. 111, 875 (2002).

[14] P. J. Westervelt, J. Acoust. Soc. Am. 25, 60 (1953).

[15] W. L. Nyborg, J. Acoust. Soc. Am. 25, 68 (1953).

[16] S. J. Lighthill, J. Sound Vib. 61, 391 (1978).

[17] C. Eckart, Phys. Rev. 73, 68 (1948).

[18] L. Rayleigh, Philos. Trans. R. Soc. London 175, 1 (1884).

[19] H. Schlichting and K. Gersten, Boundary-Layer Theory (Springer Nature, New York, 2017).

[20] W. L. Nyborg, J. Acoust. Soc. Am. 30, 329 (1958).

[21] N. Riley, Theoretical and Computational Fluid Dynamics, Vol. 10 (Springer US, Boston, MA, 1998), pp. 349-356.

[22] L. Rayleigh, The Theory of Sound (Dover, London, 2013), Vol. 1, p. 985.

[23] E. N. Da Costa Andrade, Proc. Roy. Soc. A 134, 445 (1931).

[24] J. M. Valverde, Contemp. Phys. 56, 338 (2015).

[25] M. F. Hamilton, Y. A. Ilinskii, and E. Zabolotskaya, J. Acoust. Soc. Am. 113, 153 (2002).

[26] M. Wiklund, R. Green, and M. Ohlin, Lab Chip 12, 2438 (2012).

[27] D. Ahmed, X. Mao, B. K. Juluri, and T. J. Huang, Microfluid. Nanofluid. 7, 727 (2009).

[28] X. Lu, K. Zhao, H. Peng, H. Li, and W. Liu, Phys. Rev. Appl. 11, 044064 (2019).

[29] J. Lei, M. Hill, C. P. de León Albarrán, and P. Glynne-Jones, Microfluid. Nanofluid. 22, 140 (2018).

[30] S. Subbotin, V. Kozlov, and M. Shiryaeva, Phys. Fluids 31, 103604 (2019).
[31] E. A. Jannesar and H. Hamzehpour, Arch. Acoust. 45, 35 (2020).

[32] J. Lei, M. Hill, and P. Glynne-Jones, Phys. Rev. Appl. 8, 014018 (2017).

[33] P. H. Huang, Y. Xie, D. Ahmed, J. Rufo, N. Nama, Y. Chen, C. Y. Chan, and T. J. Huang, Lab Chip 13, 3847 (2013).

[34] P. H. Huang, N. Nama, Z. Mao, P. Li, J. Rufo, Y. Chen, Y. Xie, C. H. Wei, L. Wang, and T. J. Huang, Lab Chip 14, 4319 (2014).

[35] N. Nama, P. H. Huang, T. J. Huang, and F. Costanzo, Lab Chip 14, 2824 (2014).

[36] N. Nama, P. H. Huang, T. J. Huang, and F. Costanzo, Biomicrofluidics 10, 024124 (2016).

[37] C. Zhang, X. Guo, P. Brunet, M. Costalonga, and L. Royon, Microfluid. Nanofluid. 23, 104 (2019).

[38] P. H. Huang, C. Y. Chan, P. Li, Y. Wang, N. Nama, H. Bachman, and T. J. Huang, Lab Chip 18, 1411 (2018).

[39] I. Leibacher, P. Hahn, and J. Dual, Microfluid. Nanofluid. 19, 923 (2015).

[40] Z. Cao and C. Lu, Anal. Chem. 88, 1965 (2016).

[41] H. Bachman, P. H. Huang, S. Zhao, S. Yang, P. Zhang, H. Fu, and T. J. Huang, Lab Chip 18, 433 (2018).

[42] M. Ovchinnikov, J. Zhou, and S. Yalamanchili, J. Acoust. Soc. Am. 136, 22 (2014).

[43] A. A. Doinikov, M. S. Gerlt, A. Pavlic, and J. Dual, Microfluid. Nanofluid. 24, 32 (2020).

[44] A. A. Doinikov, M. S. Gerlt, and J. Dual, Phys. Rev. Lett. 124, 154501 (2020).

[45] C. Zhang, X. Guo, L. Royon, and P. Brunet, Micromachines 11, 607 (2020).

[46] H. Bruus, Lab Chip 12, 20 (2012).

[47] S. Sadhal, Lab Chip 12, 2292 (2012).

[48] S. Boluriaan and P. Morris, Int. J. Aeroacoust. 2, 255 (2003).

[49] J. Lei, P. Glynne-Jones, and M. Hill, Microfluid. Nanofluid. 21, 23 (2017).

[50] COMSOL Multiphysics v. 5.3, COMSOL AB, Stockholm, Sweden.

[51] M. Costalonga, P. Brunet, and H. Peerhossaini, Phys. Fluids 27, 013101 (2015).

[52] S. A. Bahrani, N. Périnet, M. Costalonga, L. Royon, and P. Brunet, Exp. Fluids 61, 91 (2020).

[53] X. Guo, Y. Fan, and L. Luo, Chem. Eng. J. 227, 116 (2013).

[54] C. Zhang, P. Brunet, L. Royon, and X. Guo (unpublished).

[55] P. B. Muller and H. Bruus, Phys. Rev. E 92, 063018 (2015). 